\title{
GSA Guide to Specifying Interoperable Building Automation and Control Systems Using ANSI/ASHRAE Standard 135-1995, BACnet
}

Steven T. Bushby

Building and Fire Research Laboratory

Gaithersburg, MD

H. Michael Newman

Cornell University

Ithaca, NY

Martin A. Applebaum

ESS Engineering, Inc.

Tempe, AZ

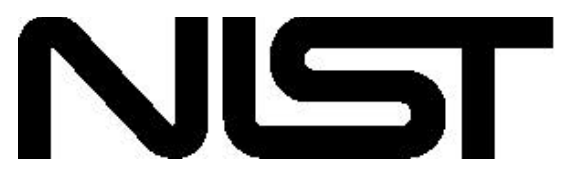

United States Department of Commerce

Technology Administration

National Institute of Standards and Technology

Gaithersburg, MD 20899 


\section{GSA Guide to Specifying Interoperable Building Automation and Control Systems Using ANSI/ASHRAE Standard 135-1995, BACnet}

Steven T. Bushby

November, 1999

Building and Fire Research Laboratory

Gaithersburg, MD

H. Michael Newman

Cornell University

Ithaca, NY

Martin A. Applebaum

ESS Engineering, Inc.

Tempe, AZ
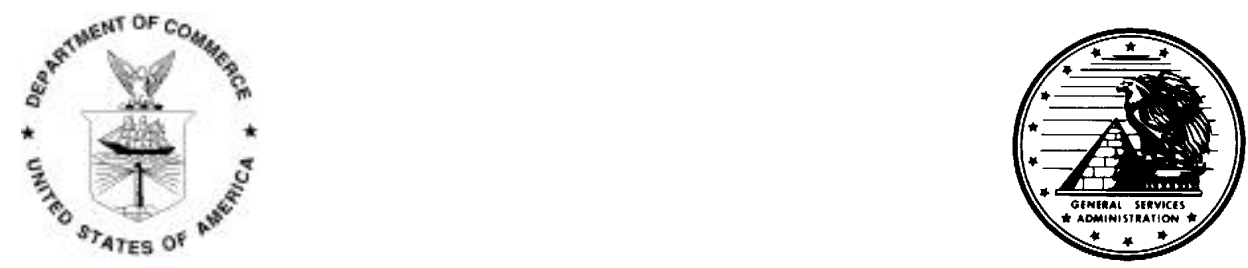

U.S. Department of Commerce

Prepared for:

William M. Daley, Secretary

U.S. General Services Administration

Technology Administration

Cheryl L. Shavers, Under Secretary for Technology

David J. Barram, Administrator

National Institute of Standards and Technology

Raymond G. Kammer, Director 


\begin{abstract}
This guide is intended for use by General Services Administration staff or design consultants when preparing specifications for interoperable building control systems using BACnet technology. It explains the issues that need to be considered when specifying the communication details and makes some recommendations for the choices involved. It is not a sample specification and it does not address all issues important for a good control system specification. The focus is on specific issues related to the interconnection and interoperation of building automation systems and devices using BACnet. It is intended to complement more general guidelines for direct digital control systems for building automation applications.
\end{abstract}

Key words: BACnet, building automation and control, direct digital control, energy management systems, communication protocol, ANSI/ASHRAE Standard 135

\title{
Acknowledgments
}

The authors wish to acknowledge the significant contributions to this guide made by the members of ASHRAE SSPC 135, the "BACnet Committee." Many of the ideas presented had their genesis in discussions with committee members about ways to make it easier to specify BACnet systems. 


\section{Table of Contents}

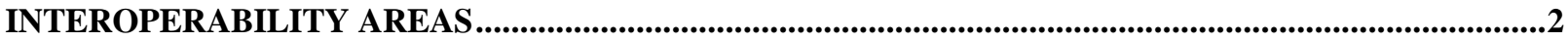

3.1 DATA SHARING

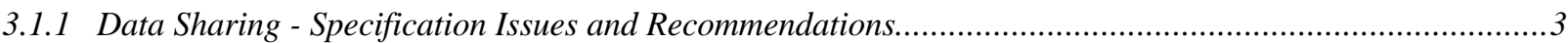

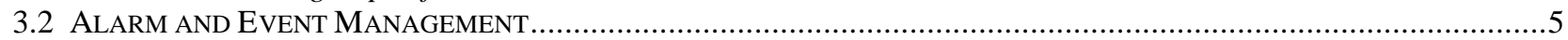

3.2.1 Alarm and Event Management - Specification Issues and Recommendations........................................5

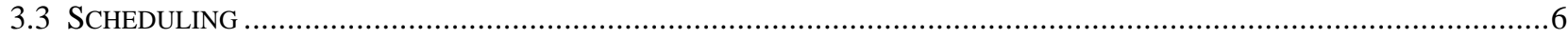

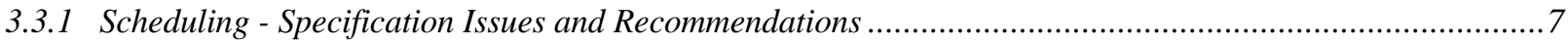

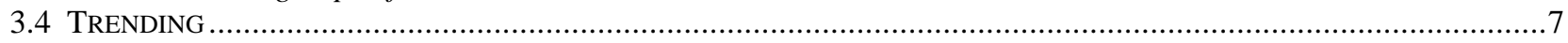

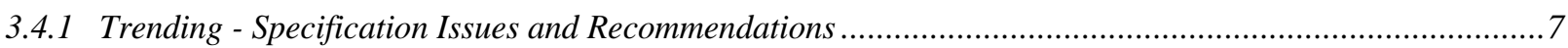

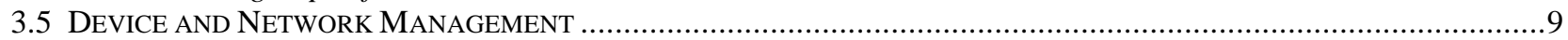

3.5.1 Device and Network Management - Specification Issues and Recommendations ....................................9

$4 \quad$ USE OF BACNET OBJECTS............................................................................................................................10

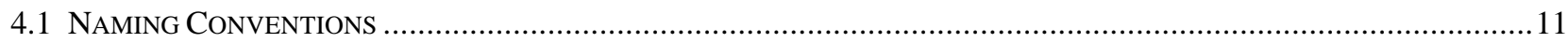

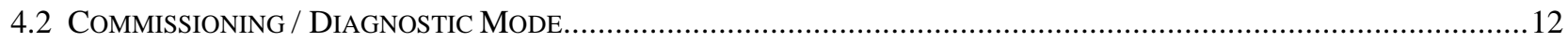

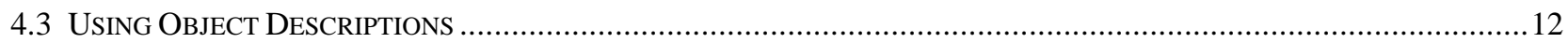

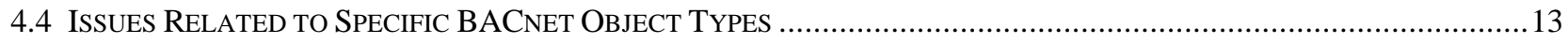

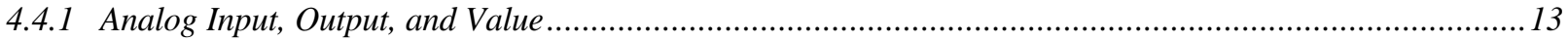

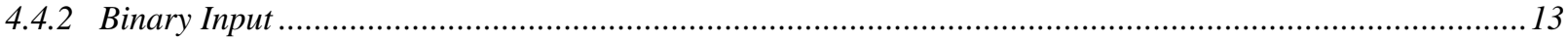

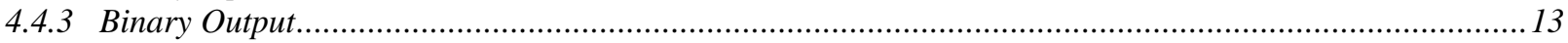

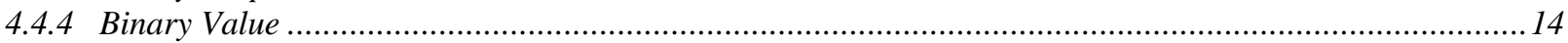

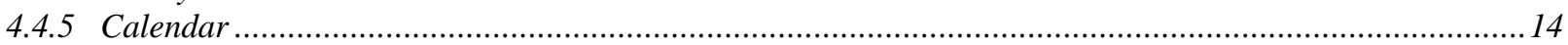

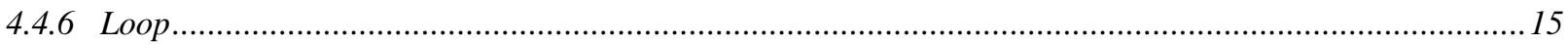

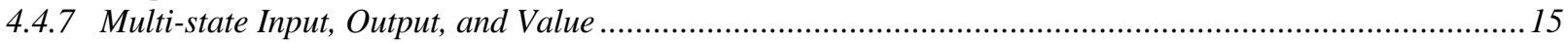

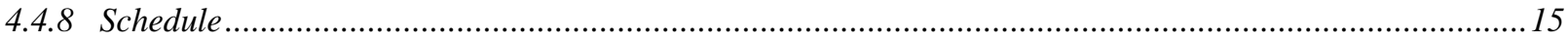

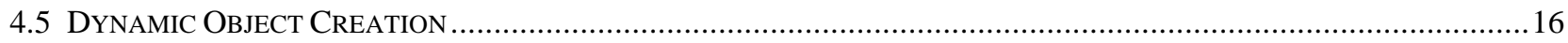

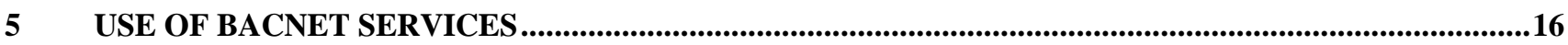

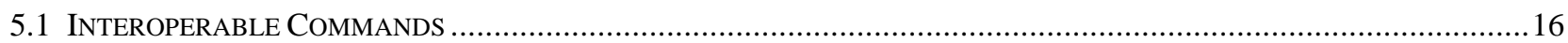

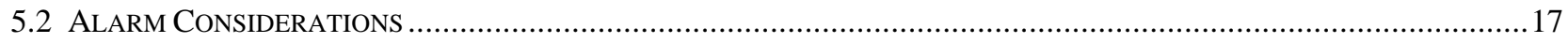

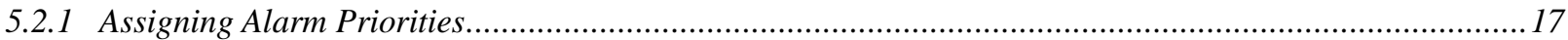

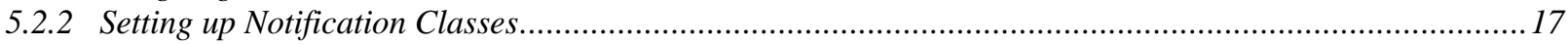

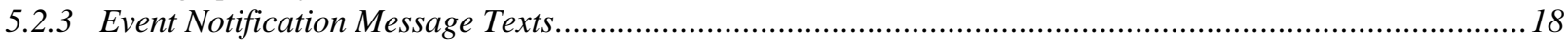

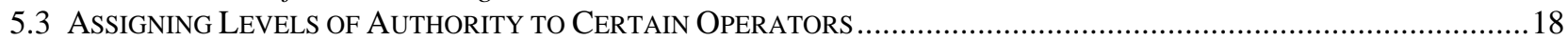

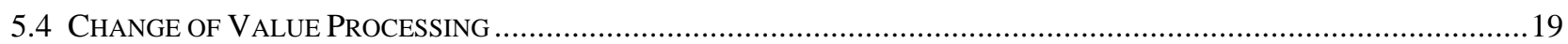

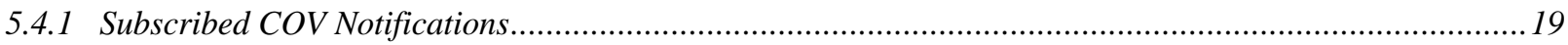

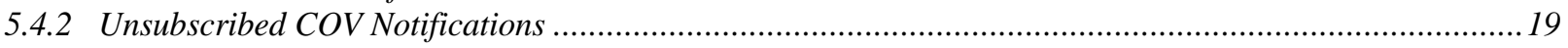

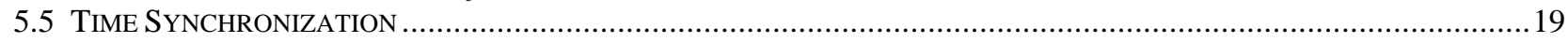

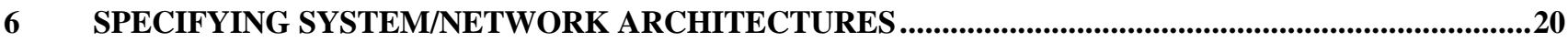

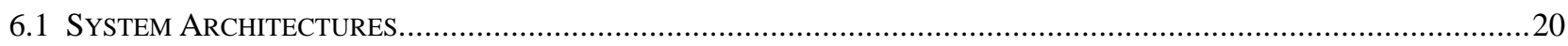

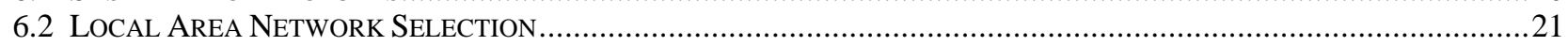

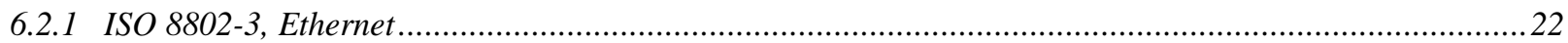

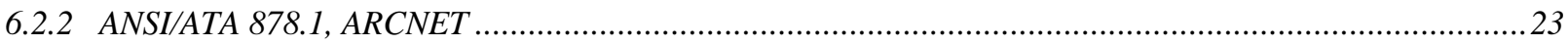

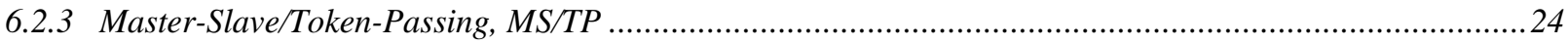

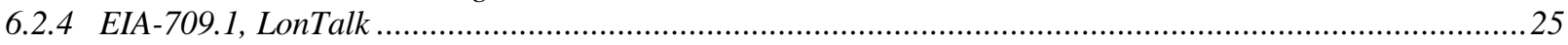

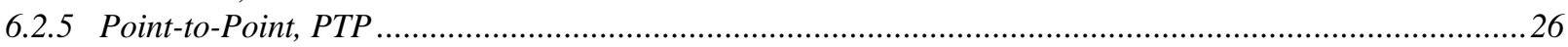

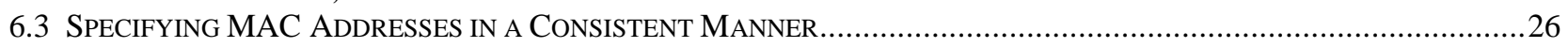




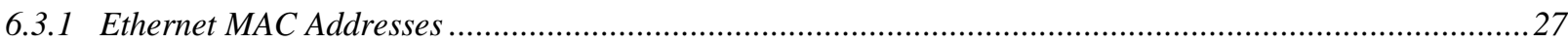

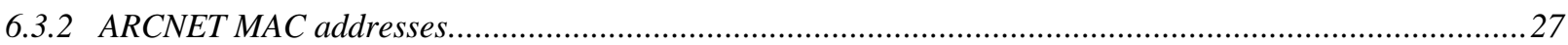

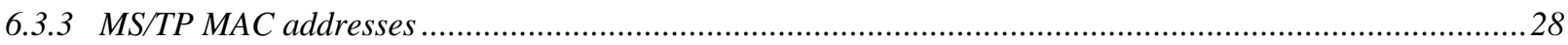

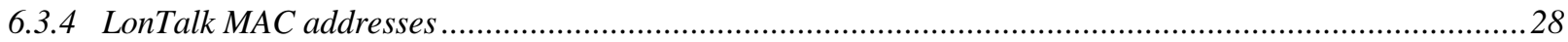

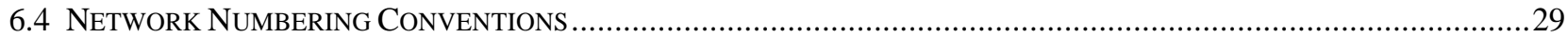

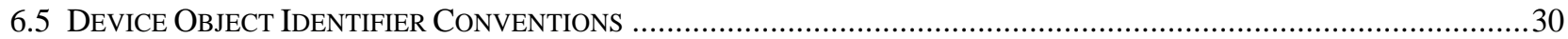

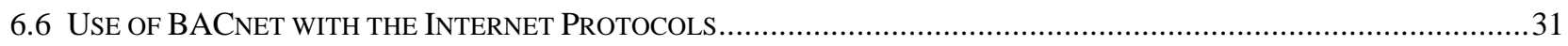

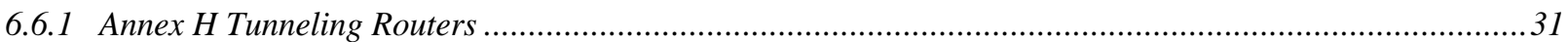

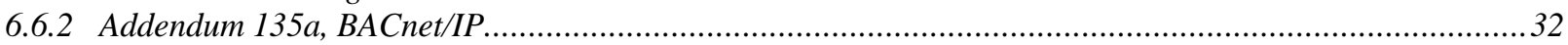

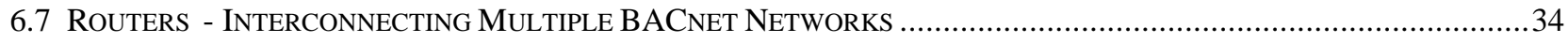

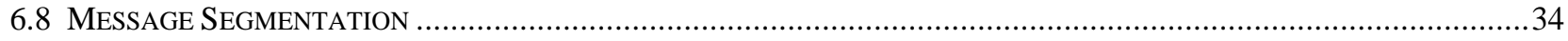

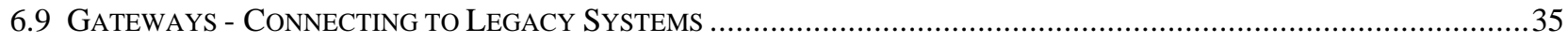

7 PRACTICAL CONSIDERATIONS IN THE CONSTRUCTION OF BACNET SYSTEMS...........................37

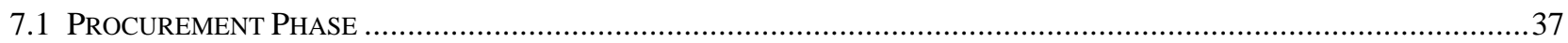

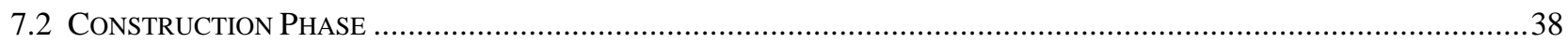

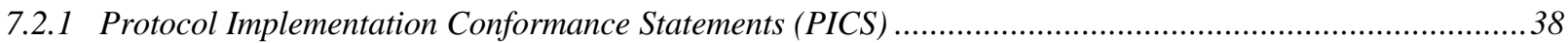

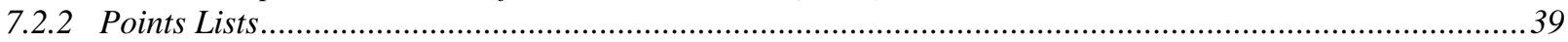

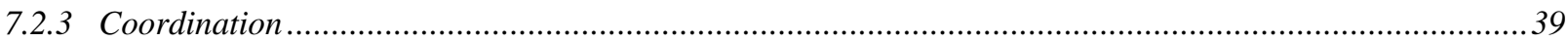

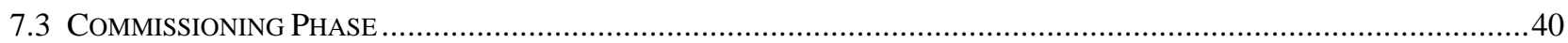

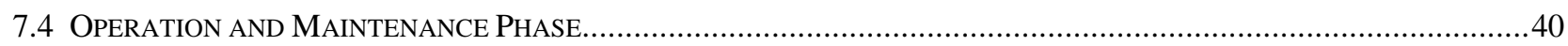

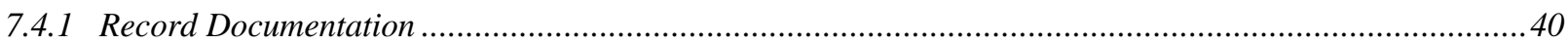

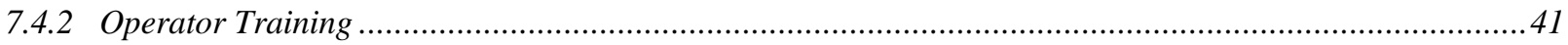

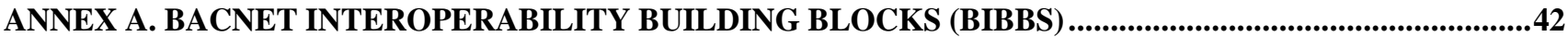

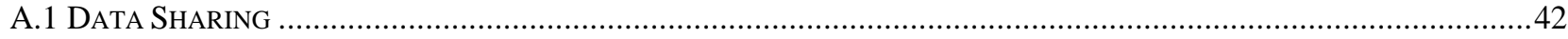

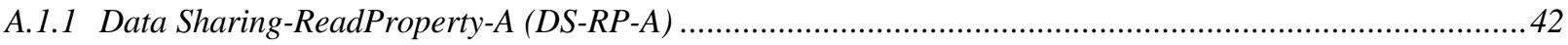

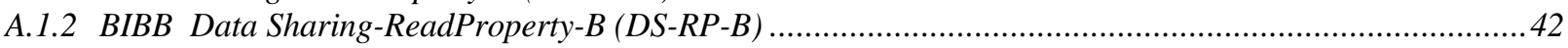

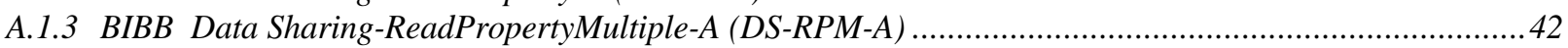

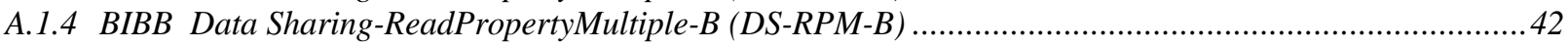

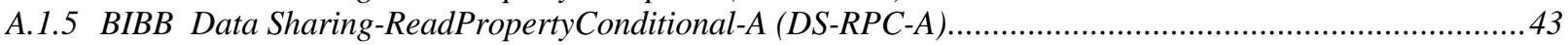

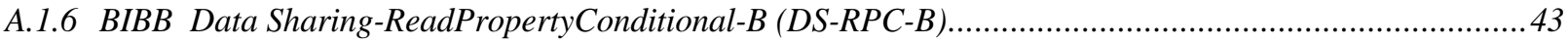

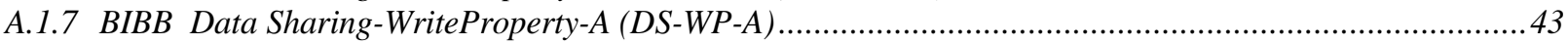

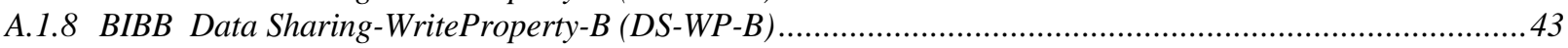

A.1.9 BIBB Data Sharing-WritePropertyMultiple-A (DS-WPM-A) ..........................................................

A.1.10 BIBB Data Sharing-WritePropertyMultiple-B (DS-WPM-B) .........................................................4

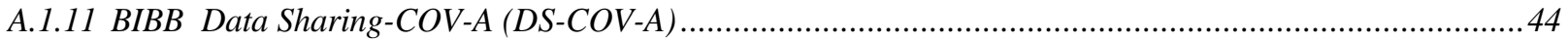

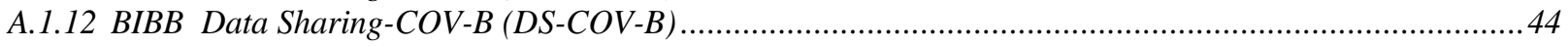

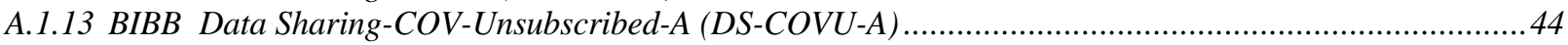

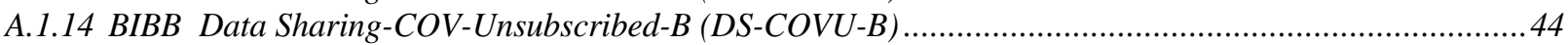

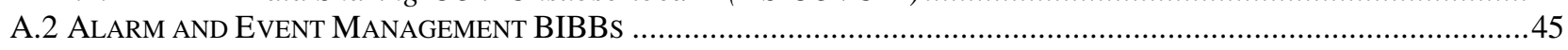

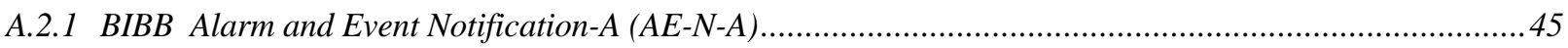

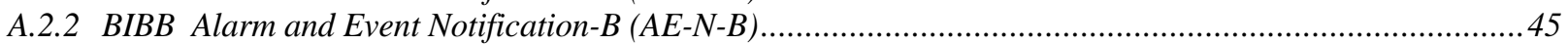

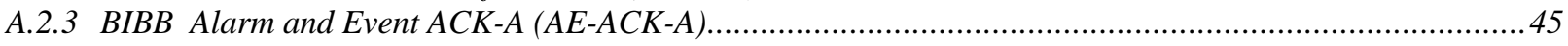

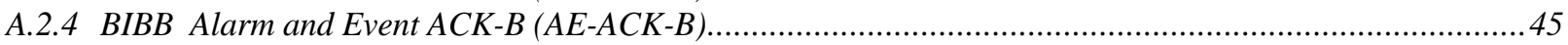

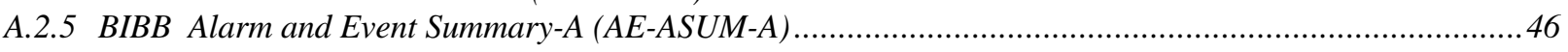

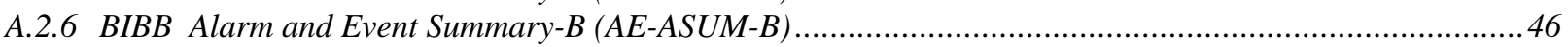

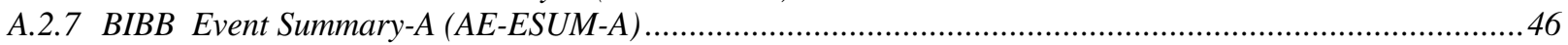

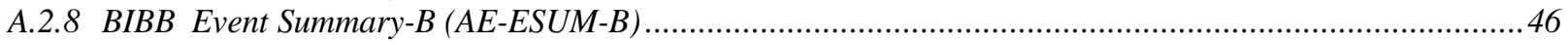

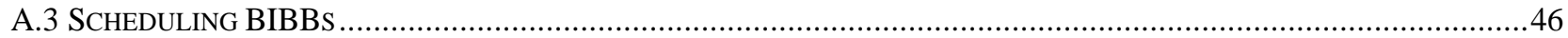

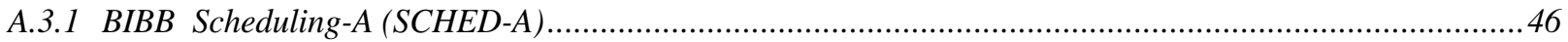

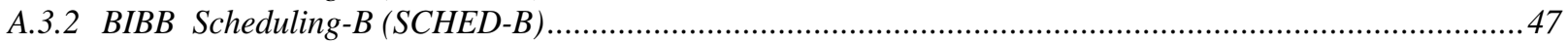

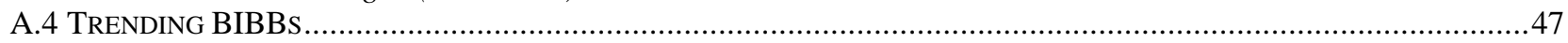

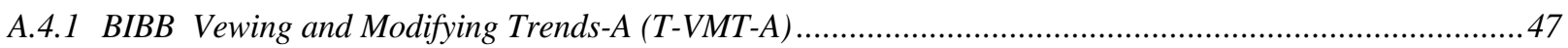

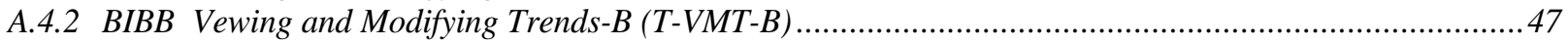




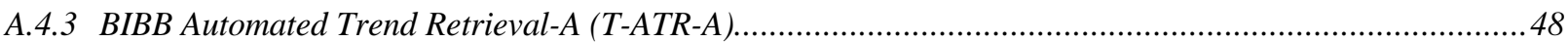

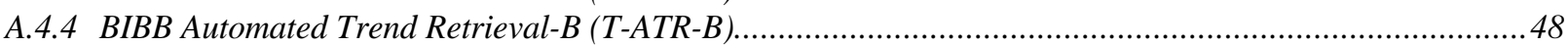

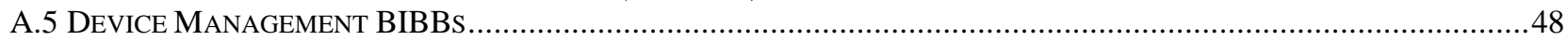

A.5.1 BIBB Device Management - Dynamic Device Binding-A (DM-DDB-A) ..............................................48

A.5.2 BIBB Device Management - Dynamic Device Binding-B (DM-DDB-B) .............................................49

A.5.3 BIBB Device Management - Dynamic Object Binding-A (DM-DOB-A) .................................................49

A.5.4 BIBB Device Management - Dynamic Object Binding-B (DM-DOB-B)..............................................49

A.5.5 BIBB Device Management - DeviceCommunication Control-A (DM-DCC-A) .............................................49

A.5.6 BIBB Device Management - DeviceCommunicationControl-B (DM-DCC-B) ........................................49

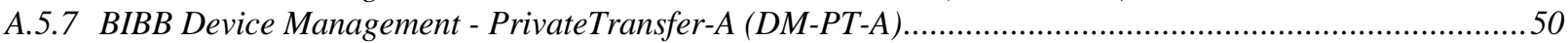

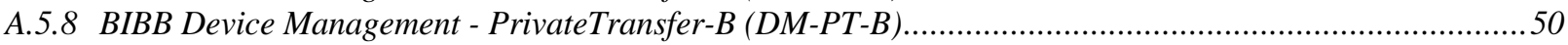

A.5.9 BIBB Device Management - Text Message-A (DM-TM-A) ...............................................................5

A.5.10 BIBB Device Management - Text Message-B (DM-TM-B) …...........................................................50

A.5.11 BIBB Device Management - TimeSynchronization-A (DM-TS-A) ..................................................50

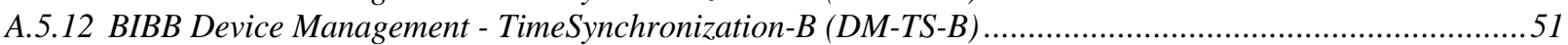

A.5.13 BIBB Device Management - UTCTimeSynchronization-A (DM-UTC-A) …........................................51

A.5.14 BIBB Device Management - UTCTimeSynchronization-B (DM-UTC-B) ….......................................51

A.5.15 BIBB Device Management - ReinitializeDevice-A (DM-RD-A)......................................................51

A.5.16 BIBB Device Management - ReinitializeDevice-B (DM-RD-B)........................................................52

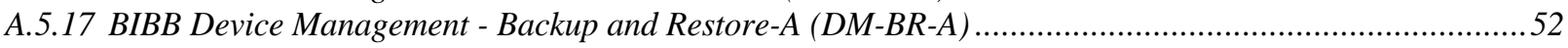

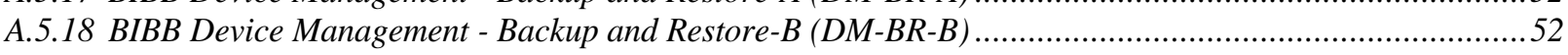

A.5.19 BIBB Device Management - List Manipulation-A (DM-LM-A) ........................................................52

A.5.20 BIBB Device Management - List Manipulation-B (DM-LM-B) .......................................................53

A.5.21 BIBB Device Management - Object Creation and Deletion -A (DM-OCD-A) .....................................53

A.5.22 BIBB Device Management - Object Creation and Deletion -B (DM-OCD-B) ....................................53

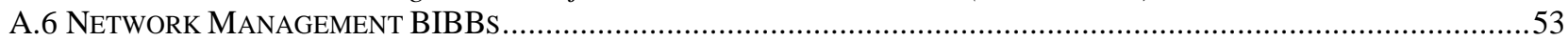

A.6.1 BIBB Network Management - Connection Establishment -A (NM-CE-A).............................................53

A.6.2 BIBB Network Management - Connection Establishment -B (NM-CE-B)................................................54

A.6.3 BIBB Network Management - Router Configuration -A (NM-CE-A) .....................................................5

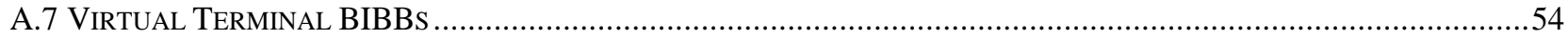

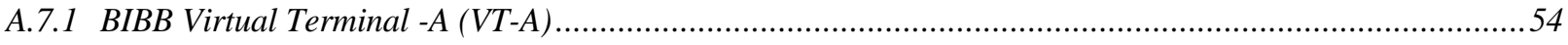

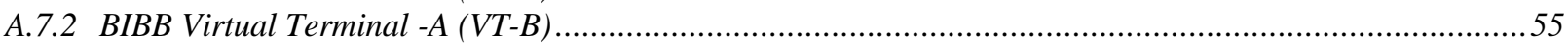

ANNEX B. DESCRIPTIONS AND PROFILES OF STANDARDIZED BACNET DEVICES................................56

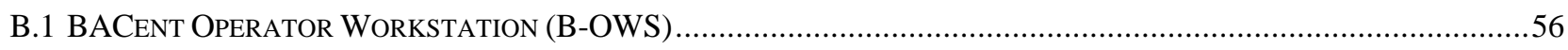

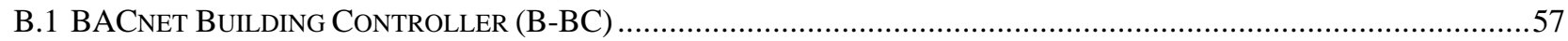

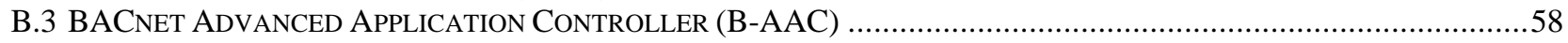

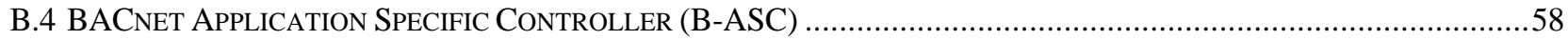

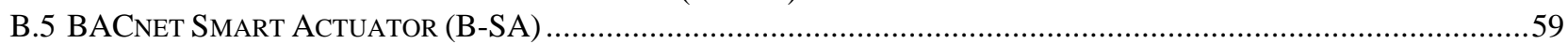

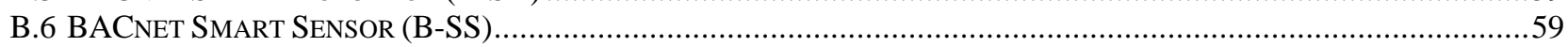

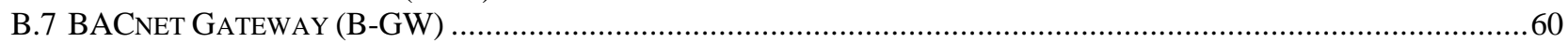

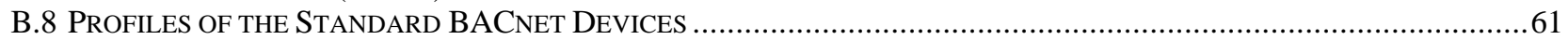

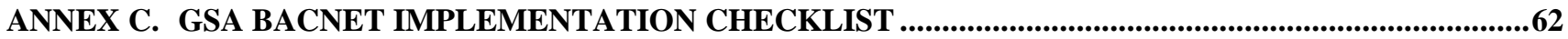




\section{Purpose of this Guide}

This document has been produced for the General Services Administration (GSA) to foster a broad-based understanding and consistent application of ASHRAE/ANSI Standard 135-1995 (BACnet ${ }^{\mathrm{TM}}$ ) for automation systems in Federal buildings - in both new and retrofit construction. As such, GSA personnel and GSA's selected design consultants should utilize this document as a guide in planning, designing, procuring and managing projects involving Building Automation System (BAS) installations, upgrades, or expansions.

As used in this guide, a BAS is a network of microprocessor-based devices that are configured to perform energy management and monitoring, heating, ventilating and air-conditioning (HVAC) control, fire protection, security, and/or related building automation functions. It is the GSA's goal to competitively procure BAS solutions that cost-effectively meet the design objectives of each project while incorporating a common BAS communication protocol to the greatest extent practicable. This then provides a foundation for non-proprietary future enhancements and expansion at each building - as well as regional/national intercommunications and information sharing across the GSA building stock. As such, all BAS projects - to some reasonable minimum degree - must comply with the BACnet standard.

It must be stressed that this guide is focused on the BACnet protocol itself, considerations for its inclusion in a BAS design, and some practical recommendations to follow in the various steps of a project that includes BAS technology. This guide is not intended to provide the details of how to specify automation systems for a particular building, HVAC applications, or other projectspecific functions. Details concerning sensors, actuators, wiring, piping, sequences of operation, and general design practice are outside the scope of this document. This guide focuses on the specific issues related to the interconnection and interoperation of BAS installations and systemlevel devices using BACnet.

For information on general BAS design issues, the reader is referred to ASHRAE Guideline 13P, Guideline to Specifying DDC Systems ${ }^{1}$. In addition, any BAS design should be prepared in compliance with applicable GSA guides for design and specification of temperature control systems (e.g., Chapter 5 of PBS-PQ100.1 "Facilities Standards" and "U.S. Courts Design Guide").

\section{Specifying Interoperable BACnet Systems}

BACnet, ASHRAE's Building Automation and Control Networking protocol, was designed to provide a single, uniform standard for building control systems. The ultimate goal of this standardization effort was "interoperability." Interoperability means the ability of disparate control system devices to work together toward a common objective through the digital exchange of relevant information. Although interoperability is often thought of in terms of interconnecting equipment from multiple manufacturers, it is also possible to contemplate interoperating systems from a single vendor, possibly equipment of different vintages. Thus, while BACnet enables multivendor interoperability, it in no way requires it.

\footnotetext{
${ }^{1}$ Available from the American Society of Heating, Refrigerating \& Air-Conditioning Engineers, Inc., Atlanta, GA. 
BACnet also provides a rich variety of tools to accomplish its communication objectives. Because of this, interoperability depends on the common selection of choices where multiple techniques are provided to accomplish a particular function. One of this guide's primary goals is to provide GSA specifiers with the information needed to understand and make the appropriate choices.

The basic strategy for specifying interoperable systems based on BACnet may be summarized in the following steps.

1. Specify the desired building automation and control system functionality. Specify the features described in the Interoperability Areas defined below as appropriate to the project.

2. Specify the desired workstation functionality. Specify the features described in the Interoperability Areas defined below as appropriate to the project.

3. Specify the desired local or wide area network technology as appropriate to the project.

4. Specify that all networks shall make use of the BACnet protocol and that all devices supplied shall implement the BACnet functionality enumerated in the device profiles in Annex B of this document.

5. Specify any additional BACnet functionality as recommended for specific requirements in this document.

\section{Interoperability Areas}

"Interoperability areas" (IAs) are intended to describe the functionality that is important in practical automation and control systems to achieve specific operational objectives. The five IAs are: data sharing, alarm and event management, scheduling, trending, and device and network management. Each IA implies a set of capabilities. Each capability, in turn, requires that specific elements of BACnet be implemented in a particular device to enable interoperability in a known and predictable manner with a minimum of field engineering. The selection of which BACnet elements are required for a particular type of device - workstation, building controller, or application specific controller - is indicated in the recommended device profiles presented in Annex B. This section describes the specific capabilities associated with each IA and the specification issues that should be considered for each IA.

Note that the original BACnet standard (ANSI/ASHRAE 135-1995) contained a clause that attempted to facilitate the specification of BACnet systems by defining "Conformance Classes" and "Functional Groups." Conformance Classes were a numerical hierarchy (1 to 6) that prescribed an increasingly complex set of BACnet capabilities intended to correspond to increasingly sophisticated control system devices. Functional Groups were additional collections of BACnet capabilities thought to be required to carry out a particular task. The idea was that a device would be designed to fall within a particular conformance class but that functional group capabilities could be added as deemed necessary for practical application. These concepts, while well-intentioned, were flawed in that the combined conformance classes and functional groups did not correspond particularly well to real-world devices in the marketplace. The scheme also required an undue amount of knowledge about the details of the BACnet specification on the part 
of the building control system design community. For these reasons, a new approach, the definition of broad areas in which interoperability is desired, has been undertaken and will form the basis of this guide.

This new approach attempts to decouple the task of the specifying engineer from that of the control system manufacturer. From the perspective of the specifying engineer, a BACnet system is described in terms of the desired building control system functions without regard for, or a need to know about, the underlying BACnet capabilities needed to carry them out. These functions, in turn, imply a set of BACnet capabilities that manufacturers can then embed in each of their devices of a particular type. The specification of these minimum requirements is the subject of ongoing work within the ASHRAE BACnet committee. The mechanism is called "BACnet Interoperability Building Blocks" (BIBBs). These are presented in Annex A.

\subsection{Data Sharing}

"Data sharing" is the exchange of information between BACnet devices. It may be unidirectional or bi-directional. Interoperability in this area permits the collection of data for archival storage, graphics, and reports, the sharing of common sensor or calculated values between devices, carrying out interlocked control strategies, and the modification of setpoints or other operational parameters of BACnet objects.

\subsubsection{Data Sharing - Specification Issues and Recommendations}

Point list

In order for suppliers to size their systems correctly in terms of their data sharing capabilities, a list of each desired data point should be provided.

- Specify as part of the fundamental system design each analog and binary input, output, and value which is to be accessed over the network.

Presentation of data

Most data are presented in the form of tabular reports, real-time graphics, or plots of data values versus time.

- Specify the desired format of all tabular reports. Indicate which point values, or sets of point values, should be available in a single report.

- Specify which building systems should be available as graphic displays. Specify the desired update interval for data on the graphics. Consider values of $1 \mathrm{~s}$ to $5 \mathrm{~s}$.

- Specify that any data value from any networked device should be available for plotting in real-time. (For the long-term archival storage of values, see the discussion of trending in 3.4) It should be possible to plot binary and analog data concurrently as well as multiple instances of each datatype on the same screen. Specify the desired sampling interval. Fast processes, such as steam valve operation, should allow rapid sampling (1s to 5 s intervals), while slow 
processes, such as space temperature monitoring, can be sampled infrequently (30s to 60s intervals). Alternatively, if the data reside in devices that support change of value reporting (see 5.4) this mechanism could be specified in lieu of a specific sampling interval.

Monitoring of any property of any BACnet object type

It should be possible to monitor any property of any standard or proprietary BACnet object type.

- Specify that it must be possible to read and display the value of any property, including all required properties, supported optional properties, and proprietary extensions of every object of every networked device.

Global objects

Some objects represent globally significant data. Examples are shared sensors, such as used to monitor the outside air temperature, or common binary information such as whether night setback is in effect.

- Specify which data are to be shared and with which devices or systems. For each piece of global information, specify the frequency of the sharing, e.g., once a minute, once an hour, once a day, or upon change of value. If change of value notifications are desired, specify the amount of change required before a new notification is transmitted.

Setpoint and parameter modification

An operator with sufficient privilege should be able to change or "write" any desired setpoint or parameter in any networked device. Since some devices may be preconfigured and their operating parameters stored in non-volatile, non-writable memory it is prudent to indicate which setpoints and parameters must be adjustable over the network.

- Specify which operating setpoints and parameters, at a minimum, must be available for modification via BACnet services (as opposed to proprietary vendor-specific means).

- Specify the desired means of making the modifications, e.g., via a graphical user interface (GUI) (as opposed to having to make a command line entry or downloading a configuration file).

\section{Peer-to-Peer data exchange}

BACnet provides the capability for information to be exchanged between networked devices without operator involvement. However, it is important to describe the required functionality clearly. 
- Specify any required data dependencies (interlocks, shared setpoints, schedules, and so on) that must be implemented via the network.

\subsection{Alarm and Event Management}

"Alarm and event management" is the exchange of data between BACnet devices related to the occurrence of a predefined condition that meets specific criteria. Such conditions are called "events" and may be the basis for the initiation of a particular control action in response or the simple logging of the event's occurrence. The event may also be deemed to represent a condition which constitutes an "alarm" requiring human acknowledgment and intervention. Interoperability in this area permits the annunciation and acknowledgment of alarms; the display of data indicating the basis for the alarm annunciation; the sharing of events for the purpose of logging or distributed control applications; modification of alarm limits and routing; and the production of summaries of the occurrence of such alarms and events.

BACnet defines two different mechanisms for generating alarms and events. One is called "intrinsic reporting" because it relies on the use of properties that are part of or "intrinsic" to the object that is being monitored for alarms or events. The other mechanism is called "algorithmic change reporting." Algorithmic change reporting is more general but is also requires the overhead of an additional object called the Event Enrollment object. The intrinsic reporting method is preferred under circumstances where it meets the objectives of the intended application.

\subsubsection{Alarm and Event Management - Specification Issues and Recommendations}

Alarm lists, operator notification and presentation of event information

The nature of each desired alarm condition and the way in which operators are notified about alarms and events should be clearly described.

- Specify all desired alarms on a system-by-system basis, including alarm limits, interlocks to avoid "nuisance" alarms (e.g., no temperature alarms when fan system is not running or during start-up or shut-down transitions), and desired response time from the occurrence of an event until a notification is provided.

- Specify how alarms are to be categorized and distributed. This will be discussed more fully below.

- Specify how alarms are to appear at the operator workstation.

- Specify that intrinsic reporting shall be used when it is sufficient to meet the functional requirements.

Alarm acknowledgment

It may be important to know which operator acknowledged an alarm and when. 
- Specify that alarm acknowledgment capability be provided and that a log be maintained that records when an alarm notification was received, when it was acknowledged, and by whom.

\section{Alarm summarization}

It should be possible, at any time, to determine the status of all defined alarms.

- Specify that it shall be possible for an operator to receive, at any time, a summary of all alarms that are currently in effect and whether or not they have been acknowledged.

- Specify that is shall also be possible to receive a summary of all alarms regardless of acknowledgment status; for which a particular recipient is enrolled for notification; based on current event state; based on the particular BACnet event algorithm (e.g., change of value, change of state, out of range, and so on); alarm priority; and notification class. These concepts will be discussed more fully below.

Alarm parameter adjustment

It should be possible for an operator to modify the alarm limits, in those cases where an alarm is based on a value exceeding some predefined limit value, or other parameters used in computing that an alarm has occurred.

- Specify that operators shall be provided the capability to dynamically modify the alarm parameters for all standard BACnet event types.

Alarm routing adjustment

BACnet provides the capability to direct alarms to different destinations based on the type and priority of the alarm, the day of week, the time of day, and so on.

- Specify that operators shall have the ability to change alarm routing for each alarm including the destination for each type of alarm and alarm priority, the day of week and time of day, and the type of transition involved (TOOFFNORMAL, TO-NORMAL and so on).

\subsection{Scheduling}

"Scheduling" is the exchange of data between BACnet devices related to the establishment and maintenance of dates and times at which specified output actions are to be taken. Interoperability in this area permits the use of date and time schedules for starting and stopping equipment and changing control setpoints as well as other analog or binary parameters. 


\subsubsection{Scheduling - Specification Issues and Recommendations}

Schedule list

In order for suppliers to size their systems correctly in terms of their scheduling capabilities, a list of scheduled activities should be provided.

- Specify each action that should take place based on the date and time of day. This should include each start/stop operation and each change of operating setpoints and parameters based on operating mode, e.g., night set-back, vacation and holiday operation, special event operation, and so on.

Display of start and stop times of scheduled devices

BACnet provides the capability to implement control actions based on dates and times. These actions can cause devices to start and stop or setpoints or other analog parameters to be modified. It is important for an operator to be able to ascertain what actions are scheduled for any given date and time.

- Specify that an operator shall be able to inspect the content of any schedule and determine the specific control actions that will occur at any time, on any date. Additionally, for any particular device or system parameter that is the subject of a schedule an operator shall be able to determine the schedule of actions related to that device or parameter.

Modification of schedules

Although BACnet defines the operation of calendars and schedules, it does not require that they be modifiable in all cases over the network. If this is needed, it should be definitively specified.

- Specify that certain, or all, calendar entries and schedules shall be modifiable from an operator workstation.

\subsection{Trending}

"Trending" is the accumulation of (time, value) pairs at specified rates for a specified duration. The values are those of a specific property of a specific object. "Trending" is distinguished from the real-time plotting of data (see 3.1.1) in that the data are usually destined for long-term storage and the sampling intervals are usually longer. Interoperability in this area permits the establishment of trending parameters and the subsequent retrieval and storage of trend data.

\subsubsection{Trending - Specification Issues and Recommendations}

Archival storage of data

It should be possible to archive data values from any networked device but the supplier needs to be able to calculate the required disk storage. 
- Specify the maximum number of data points for which archival storage is likely to be desired. A distinction should be made between points with true historical value, e.g., energy use, and those that only need to be trended for a short period of time such as points used to verify system operation following a repair or retrofit.

- Specify the minimum sampling interval required. For long-term trending, a rate of 5-6 samples per hour will often suffice.

- Specify the duration of time for which the archived information must be available for on-line retrieval. One to two years will often be adequate.

- Specify the means for archiving older data. A reliable tape system or writable CD would be appropriate.

Trend list

In order for suppliers to size their systems correctly in terms of their trending capabilities, a list of initially required trend logs should be provided.

- Specify the initial requirements for trending in terms of which data points are to be trended, the sampling rate, the duration of each trend log, and the length of time it is desired to keep the resulting $\operatorname{logs}$ available on-line. Alternatively, specify the approximate number of desired trend logs along with other parameters.

Display and archive of trend data

BACnet provides the capability for field devices to collect data and then notify a workstation or storage device that a trend log is available for retrieval.

- Specify that an operator will be able to retrieve and display trend logs, access the underlying numerical data in spreadsheet format, and output the data to printers or other files.

Modification of trend $\log$ parameters

Operators with sufficient privilege should be able to modify the trend log parameters online from any BACnet workstation.

- Specify that the data points to be logged, the sampling rate, and duration of trend $\log s$ shall be modifiable by an authorized operator from a system workstation. 


\subsection{Device and Network Management}

"Device and network management" is the exchange of data between BACnet devices concerning the operation and status of specific devices. Interoperability in this area permits determining which devices are present on a given network and some of their operational capabilities, including which objects they maintain; the ability to start up and shut down communication from a particular device; the ability to synchronize the time in those devices that maintain clocks; the ability to reinitialize the operation of a device's computer; the ability to establish connections as needed; and the ability to change the connection configuration.

\subsubsection{Device and Network Management - Specification Issues and Recommendations}

Display of information about device status

An operator should be able to query the status of any device on the internetwork.

- Specify that an operator shall be able to display at any time the operational status of any device on the BACnet internetwork.

Display of information about any BACnet object

An operator should be able to display information about any BACnet object or group of BACnet objects.

- Specify that an operator shall be able to display at any time any property of any BACnet object.

- Specify that an operator shall also be able to display property values of objects grouped by object type, object location, building system, and so on.

Ability to silence a device on the network that is transmitting erroneous data

If a sensor malfunction should occur, an operator should be able to quiesce the offending device until repairs can be effected.

- Specify that an operator shall be able to direct a field device to stop transmitting event or alarm notifications until a subsequent command to resume transmissions is received.

Ability to synchronize the time in devices across the BACnet inter-network

It should be possible for an operator to issue time synchronization commands to one or more devices as needed. This capability is independent of the existence of a "time master" on the network that carries out time synchronizations automatically.

- Specify that an operator shall be able to set the time and date in any device on the network that supports time-of-day functionality. This capability should be 
provided for both individual devices or groups of devices, including all devices simultaneously.

Ability to cause a remote device to reinitialize itself

BACnet provides the capability to issue a command that will cause a remote device to "reinitialize" itself. This usually amounts to restarting or rebooting the computer itself and starting any control activities "from scratch."

- Specify that an operator shall have the ability to issue reinitialization commands to any device that supports remote reinitialization.

Ability to backup and restore the configuration of other devices

BACnet provides the ability for devices to be backed up and restored over the network. Although not all devices support this capability (e.g., because the operating software is ROM-based), it is still important to have this ability if available.

- Specify that an operator shall have the ability to backup and restore all BACnet devices on the network that support this capability.

Ability to command half-routers to establish and terminate connections

BACnet "half-routers" are used to provide connectivity to remote sites, usually on a temporary basis, using dial-up telephone technology.

- Specify that an operator shall have the ability to issue a command for the establishment or termination of a connection to a remote BACnet network.

Ability to query and change the configuration of half-routers and routers

Operators should have the ability to change the database entries that half-routers and routers use to establish communications with remote BACnet networks.

- Specify that an operator shall have the ability to display and modify the routing table entries in all provided BACnet half-routers and routers.

\section{Use of BACnet Objects}

BACnet objects form the basis for representing the functionality of building automation and control equipment in a standard, network-visible way. The original BACnet standard defined 18 object types and 3 more are in the final approval stage. While the use of the Interoperability Areas described above in conjunction with the device profiles of Annex A should result in devices being provided with a known set of BACnet capabilities, the number and type of BACnet objects that will be provided depends on the specificity of the desired control sequences of operation and other functional requirements of a particular installation, such as the number of alarms, trending logs, schedules, metering points, and so on. In this section a number of specification issues will be presented that are specific to BACnet objects. 


\subsection{Naming Conventions}

BACnet objects are uniquely identified within a particular BACnet device by a 32-bit numeric "object identifier." While this expedites access over the network and allows implementers to know, in advance, how much storage to allocate for these identifiers, their use by human operators would be highly impractical. Therefore, BACnet also allows each object to be referenced by an "object name." The standard, however, only specifies a minimum length of one printable character for the object name and does not even require that it be writable, i.e., modifiable, once a device is commissioned. This was done to accommodate very simple devices, such as might be employed as unitary or application specific controllers.

Both object identifiers and object names are required to be unique within the device that contains them. There is an additional constraint on the Device object that its name and object identifier be unique within the entire BACnet internetwork including any temporary dial-up connections. Except for the Device object, manufacturers can freely configure object identifiers with no adverse impact on interoperability or the expandability of the system. The special case of Device object identifiers is discussed in 6.5. Object names should be assigned according to a convention established by the facility manager and not left for the manufacturer to arbitrarily decide.

The use of object names typically comes up in two very different contexts. The first is in the programming of BACnet devices for a particular purpose where an object is referred to in the program. The second is in referring to the object from a workstation where an operator may want to view properties of a particular object or insert a property in a graphic or in a tabular report. In the latter case, the workstation will generally retain a mapping of the workstation name to the object identifier (or object name) used in the remote device.

The basic principle to follow in setting up site-wide object (and device) naming conventions is that the names should be as meaningful as possible given the name length available. Thus, "AHU1.STEMP" would be preferable to a more cryptic name like "A1ZXC5". An often used convention is to construct a name from a set of components, each of which relates to the building system installation. In this scheme the compound name is constructed from the building or facility in which the system is located, the name of the building system itself, and, finally, the name of the desired point. An example of this FACILITY.SYSTEM.POINT (FSP) naming method is "BLDG226.AHU1.MA_TEMP". The facility is Building 226, the system is Air Handler Unit 1 , and the point is the mixed air temperature. The convention may be easily extended to accommodate areas within the facility, and subsystems within a system. For example, "BLDG226.BASEMENT_MER.AHU1.CW.STEMP" would refer to the supply temperature of the chilled water subsystem of the air handler located in the basement mechanical equipment room.

- Specify a set of abbreviations for common building systems, subsystems, and points that are to be used by all vendors.

- Specify that object name properties, in those devices where the object name is configurable, shall be at least 50 characters long and shall, to the extent possible make use of a system and point nomenclature using the abbreviations specified above.

- Specify that BACnet object names used in workstations may be up to 50 characters long and shall consist of a string made up of components indicating, as appropriate, the 
location, system, subsystem, and point of the object. These object names shall be used in workstation applications such as graphics, reports, and alarms wherever an object name is appropriate in lieu of the object name property in the remote device. Moreover, an operator may display at any time the mapping between the object name used on the workstation and the object name property used in the remote BACnet device.

\subsection{Commissioning / Diagnostic Mode}

A powerful feature of the BACnet object model is the ability to verify controller performance by manipulating properties of certain objects and observing the resulting device operation. For example, the Present_Value property of an Analog Value object could be set to a value above the High_Limit and the execution of a programmed response to this condition could then be verified. This capability is of particular value during commissioning or for diagnosing problems subsequent to installation and acceptance.

In order to be able to decouple the Present_Value of an object from its underlying process and force it to take on a different value, the object must first be taken "out of service" by causing its Out_Of_Service property to be TRUE. The Out_Of_Service property is provided for the Loop, Program and all of the Analog, Binary, and Multi-state object types. In order to accommodate the requirements of very simple devices, however, BACnet does not require that the Out_Of_Service property be writable. In such devices Out_Of_Service may possibly be accessed and altered by non-BACnet means such as proprietary configuration devices or protocols. In order to be able to conduct commissioning and diagnostics using the BACnet network, it should be requested that the Out_Of_Service property be writable.

- Specify that the Out_Of_Service property shall be adjustable (writable) using BACnet services for all Analog, Binary, Multi-state, Loop and Program objects.

\subsection{Using Object Descriptions}

All BACnet object types have an optional character string Description property whose length and content are not defined or limited. The idea was to provide a mechanism to allow an operator or technician to obtain useful information about the purpose or application of a particular object in any BACnet device. The Description property is optional because text takes up a relatively large amount of memory and may exceed the resources of simple devices. Also, descriptive object information may be stored in a database external to the device in which the object resides, e.g., in a workstation, thus conserving resources in a device with limited storage.

Nonetheless, the availability of a Description property in certain objects is highly desirable. This is particularly true for the Device object. Its description can convey its location, purpose, or other significant data.

- Specify that each Device object shall have a Description property and that the length available for the Description properties of other implemented object types shall be provided in the "Property Range Restrictions" portion of the device's PICS. 


\subsection{Issues Related to Specific BACnet Object Types}

The specification should provide direction to suppliers for the values to be assigned to certain properties of BACnet objects that need to be coordinated and made consistent in the case where there may be multiple suppliers on a job. In BACnet, the values of these properties are referred to as "local matters," a term meaning that the value depends on the local circumstances.

\subsubsection{Analog Input, Output, and Value}

The analog object types have the ability to announce changes of value using the COV reporting method.

- Specify that all analog objects (Input, Output, and Value) shall have the capability of using the Change of Value reporting mechanism and that the COV_Increment property shall be writable using BACnet services.

\subsubsection{Binary Input}

Binary inputs have two properties, Inactive_Text and Active_Text, whose values should be specified.

- Specify, for each Binary Input object, the text to be used for the Inactive_Text and Active_Text properties. For example, "On", "Off"; "Running", "Stopped", "Open", "Closed", and so on.

\subsubsection{Binary Output}

In addition to Inactive_Text and Active_Text, Binary Output objects may need to have values specified for the Feedback_Value, Minimum_On_Time, and Minimum_Off_Time properties.

Binary Output objects have optional properties that are used to keep track of how many times the state has changed and to accumulate the total run time. If either of these features is appropriate for the application, support for these properties must be specified.

- Specify, for each Binary Input object, the text to be used for the Inactive_Text and Active_Text properties. In addition, if appropriate for the application, also specify appropriate values for the Feedback_Value, Minimum_On_Time, and Minimum_Off_Time properties.

- Specify support for the Change_Of_State_Time, Change_Of_State_Count, and Time_Of_State_Count_Reset if counting state changes is appropriate for the application. Also specify that Change_Of_State_Count be writable so that the count can be reset.

- Specify support for the Elapsed_Active_Time and Time_Of_Active_Time_Reset properties if accumulating run time is appropriate 
for the application. Also specify that Elapsed_Active_Time be writable so that the run time can be reset.

\subsubsection{Binary Value}

In addition to Inactive_Text and Active_Text, Binary Value objects may need to have values specified for the Minimum_On_Time, and Minimum_Off_Time properties.

Binary Value objects have optional properties that are used to keep track of how many times the state has changed and to accumulate the total run time. If either of these features is appropriate for the application support for these properties must be specified.

- Specify, for each Binary Value object, the text to be used for the Inactive_Text and Active_Text properties. In addition, if appropriate for the application, also specify appropriate values for the Minimum_On_Time, and Minimum_Off_Time properties.

- Specify support for the Change_Of_State_Time, Change_Of_State_Count, and Time_Of_State_Count_Reset if counting state changes is appropriate for the application. Also specify that Change_Of_State_Count be writable so that the count can be reset.

- Specify support for the Elapsed_Active_Time and Time_Of_Active_Time_Reset properties if accumulating run time is appropriate for the application. Also specify that Elapsed_Active_Time be writable so that the run time can be reset.

\subsubsection{Calendar}

Calendar objects store lists of dates, date ranges, or month/week/day combinations like the "first Tuesday of every month." The most typical use of a Calendar object would be to store a list of holidays, vacation periods, or special events that would be referenced by a Schedule object. BACnet does not specify how many Calendar objects should be provided, how many entries each Calendar must allow, or even that the entries be modifiable over the network.

- Specify that devices that provide scheduling capability shall also provide at least one Calendar object with a capacity of at least 10 entries. It shall be possible to view the Calendar object and make modifications from any BACnet workstation on the network.

- Specify, if the Calendar's Date_List property is writable using BACnet services, that all calendar entry datatypes must be supported. These are individual dates, date ranges, and specific weeks and days within a month. 


\subsubsection{Loop}

Loop objects provide a network visible representation of control loops including proportional-integral-derivative (PID) control loops. If it is anticipated that building engineers will want to tune such control loops from a BACnet workstation (as opposed to using proprietary tuning methods), i.e., make adjustment to the various gain, bias, and sampling parameters, then the use of Loop objects should be required.

- Specify that the desired PID control loops shall be represented by Loop objects and that the tuning constant properties shall be writable. These include the Update_Interval, Setpoint, Proportional_Constant, Integral_Constant, Derivative_Constant, and Bias. Specify writability of other Loop properties as appropriate to the application.

- Specify that all Loop objects shall have the capability of using the Change of Value reporting mechanism and that the COV_Increment property shall be writable using BACnet services.

\subsubsection{Multi-state Input, Output, and Value}

The Multi-state object types have State_Text properties that describe each of the possible states that the Present_Value of the object may take on. In addition, Multi-state Output objects may have a Feedback_Value.

- Specify, for each Multi-state Input, Output, and Value object, the text to be used for each state that the object can represent. In addition, if appropriate for the application, also specify how the Feedback_Value is to be determined.

- Specify that all Multi-state Input, Output, and Value objects shall have the capability of using the Change of Value reporting mechanism and that the COV_Increment property shall be writable using BACnet services.

\subsubsection{Schedule}

Schedule objects provide a way to alter binary or analog values based upon date and time. These values will typically be associated with physical outputs or with operational parameters such as setpoints. A single schedule may be applied to multiple data points, each of which are subject to the same value being applied at the same time, e.g., multiple fan systems, all of which are turned on at 7 A.M. and off at 6 P.M. during the work week. However, BACnet does not specify how many Schedule objects should be provided, or that any of their properties must be modifiable over the network.

- Specify each building system that is to be subjected to date and time scheduling and specify that it shall be possible to modify schedule entries from a BACnet workstation. 


\subsection{Dynamic Object Creation}

BACnet provides the ability to create new objects dynamically, i.e., after a device has been configured initially. While, in theory, new instances of any object type could be created, the primary use of this capability is the creation of Averaging, Calendar, Event Enrollment, Group, Notification Class, Schedule, and Trend Log objects.

- Specify, if required by the application, that it shall be possible to dynamically create instances of the Averaging, Calendar, Event Enrollment, Group, Notification Class, Schedule, and Trend Log objects.

\section{Use of BACnet Services}

The following sections discuss specific issues related to the use of BACnet "services," the clientserver messages that are exchanged between BACnet devices.

\subsection{Interoperable Commands}

One of the most significant aspects of BACnet is the implementation of a "command priority" scheme. This makes possible the "prioritization" of commands from various control processes, e.g., start-stop commands or setpoint changes, so that it is predictable which command will be carried out. This is implemented by assigning "command priority levels" to the various processes. BACnet prescribes a set of standard priority levels as shown in Table 1:

Table 1. Standard Command Priorities

\begin{tabular}{|c|l|c|l|}
\hline $\begin{array}{c}\text { Priority } \\
\text { Level }\end{array}$ & \multicolumn{1}{|c|}{ Application } & $\begin{array}{c}\text { Priority } \\
\text { Level }\end{array}$ & \multicolumn{1}{|c|}{ Application } \\
\hline 1 & Manual-Life Safety & 9 & Available \\
\hline 2 & Automatic-Life Safety & 10 & Available \\
\hline 3 & Available & 11 & Available \\
\hline 4 & Available & 12 & Available \\
\hline 5 & Critical Equipment Control & 13 & Available \\
\hline 6 & Minimum On/Off & 14 & Available \\
\hline 7 & Available & 15 & Available \\
\hline 8 & Manual Operator & 16 & Available \\
\hline
\end{tabular}

Note that priority levels $3,4,7$, and 1-16 are available for assignment. Thus the task for the designer becomes 1) deciding what processes need to be included in the prioritization scheme and 2) what relative importance, i.e., command priority level each should be assigned to. Examples of processes that should be considered for prioritization are: peak demand limiting, 
night set-back, night cooling, and optimum start-stop. Interoperable command priorities must be applied uniformly throughout the entire internetwork.

- Specify the processes that are to be prioritized and the command priority levels assigned to each if they do not fall into the pre-assigned levels shown in Table 1. For each point subject to the command priority scheme, specify a default status, position, or value for the point to take on in the absence of a prioritized command.

\subsection{Alarm Considerations}

This section describes design considerations relating to alarm management in BACnet systems.

\subsubsection{Assigning Alarm Priorities}

BACnet provides the ability to assign a numeric priority to each event transition for which notification is desired. The transitions are TO-OFFNORMAL, TO-FAULT, and TO-NORMAL. The meaning of each transition depends on the algorithm used to process the alarm inputs. The most common algorithms have been standardized and are precisely defined in BACnet. They are Change of Bitstring, Change of State, Change of Value, Command Failure, Floating Limit, and Out of Range alarms.

Priority values range from 0 to 255 with 0 being the highest priority and 255 the lowest. Typical installations will use only a small number of discrete values such as "255, 128, 0" for "low, medium, high" but the values to be used need to be conveyed to the installers. Events of type Alarm are generated in BACnet by objects that support "intrinsic reporting" or by Event Enrollment objects. Object types that support intrinsic reporting are the Analog Input, Output, and Value; Binary Input, Output, and Value; Multi-state Input, Output, and Value; and Loop. Intrinsic reporting is tightly prescribed in that it pertains only to an object's Present_Value or Status_Flags properties. Event Enrollment objects can apply the standard algorithm types to any property of any object.

In general, alarm distribution is managed by the use of "notification classes" which are described in next section. One of the parameters conveyed in the alarm notification is the priority value which is a property of the associated Notification Class object or the Event Enrollment object. The use to which the priority is put is not dictated by BACnet.

The task of the designer is to assign priority values to the alarms that are to be implemented and to specify the actions to be taken by recipients upon receipt. These could include directing the notification to a printer; sounding an audible or visual signal; generating a report with alarms of the same priority collected together; and so on.

- Specify the priority values to be assigned for each alarm in the system. For each priority value, or range of values, specify the actions to be taken upon receipt.

\subsubsection{Setting up Notification Classes}

As mentioned in the preceding section, BACnet provides the ability to direct alarms to different destinations based on the type of the alarm transition, the day of week, and the time of day. The 
mechanism for achieving this is to create and configure Notification Class objects, each of which is referenced by other processes as a simple integer known as the "notification class."

In the most elementary situation, there would be a single notification class, perhaps statically configured as a default, that would be valid 24 hours a day and would direct all messages to a single recipient. In the more general case, there might be multiple recipients who should only receive specific types of alarms and, perhaps, only at certain hours of the day. A building manager, for instance, might receive all alarms at all times, but only critical alarms would be sent via a pager or to the night shift operator between midnight and 8 A.M.

- Specify how alarms should be distributed by specifying the recipients of each type and priority of alarm. If desired, specify the valid days of the week and times of the day for each.

\subsubsection{Event Notification Message Texts}

The occurrence of alarms and events in BACnet is signaled by the transmission of "Event Notification" messages. These messages convey most of the information that is important about the occurrence including the "what, when, and where." There is also an optional parameter called 'Message Text'. While many current systems use workstation software to translate an alarm identifier into text meaningful to an operator, the text message may also be composed by software resident in a field device and sent along with the other alarm data. Either way, it is useful to specify the desired content and format of the alarm messages that will be seen by an operator. Apart from the alarm details, the messages can also be used to indicate any necessary actions to be taken. The messages might contain, for example, the name of a particular mechanic, service contractor, or phone number to be called, or indicate that certain other data points should be inspected to verify the validity of the alarm condition.

- Specify the content and format of the alarm messages that will be delivered to operators.

\subsection{Assigning Levels of Authority to Certain Operators}

Tracking the acknowledgment of alarms is one important aspect of facility management. BACnet devices can be configured to accept alarm acknowledgments only from specific personnel. The broader issue is the assignment, if deemed appropriate, of differing levels of operational authority to different personnel. While this is mainly an administrative issue relating to the configuration of workstation software, several BACnet services have optional password protection. They are the remote device management services, DeviceCommunicationControl and ReinitializeDevice. The first allows an operator to "quiesce" a device that is generating specious messages due, for example, to a defective sensor, and the second forces a remote device to go through a restart process. In each case, the passwords are character strings of up to twenty characters that are programmed into the remote devices.

- Specify, if desired, the number of authorization levels and the operator accounts to be assigned to each. If password protection for remote device management services is needed, specify the passwords to be configured. Alternatively, specify that there shall 
be a method provided for dynamically assigning passwords for remote device management functions after installation.

\subsection{Change of Value Processing}

A powerful BACnet capability is that of "change of value" (COV) reporting. In essence, certain standard object types can be configured to generate a notification when their present values change by a prescribed amount or their status flags (the bits that indicate an object's in-alarm, fault, overridden, and out-of-service status) change at all. The object types that possess this optional capability are the Analog, Binary, and Multi-state Input, Output, and Value, and the Loop. This type of COV notification can be useful for efficiently updating real-time graphic displays and, in terms of limiting unnecessary network traffic, is preferable to continuously reading the value of a point to detect a change of value.

\subsubsection{Subscribed COV Notifications}

In order to receive COV notifications, a subscription request must be sent to the device which contains the objects in question.

- Specify workstation software shall have the capability of subscribing to COV notifications for all object types that support it.

\subsubsection{Unsubscribed COV Notifications}

A new feature of BACnet that is currently under public review is the use of the UnconfirmedCOVNotification service as a means of distributing COV notifications for changes that have not been subscribed to. This mechanism is intended to be used for distributing shared, globally significant, values, e.g., a common outside air temperature or a binary value indicating whether a building is "occupied" or not.

- Specify that changes of value of globally shared data shall be distributed by means of UnconfirmedCOVNotifications.

\subsection{Time Synchronization}

It may be desirable to provide a standardized time reference for a single building system or group of systems. In BACnet, Time synchronization is achieved by defining a computer to serve as a "Time Master." Depending on the scope of a particular project, i.e., whether it is localized or spans time zones, it may be desirable to use "Coordinated Universal Time" (UTC) as the reference rather than local time.

- Specify that a time master shall be provided and the format of the time synchronization messages, either local time or UTC. 


\section{Specifying System/Network Architectures}

BACnet provides considerable flexibility in the selection of network types and interconnections between them. This section discusses the issues involved with deciding which network types to use and how to interconnect them.

\subsection{System Architectures}

The term "system architecture" refers to the arrangement of networks that make up an "internetwork." See Figure 6-1. Note that in BACnet, each network in an internetwork, is identified by a unique "network number." The assignment of network numbers is further discussed in 6.4.

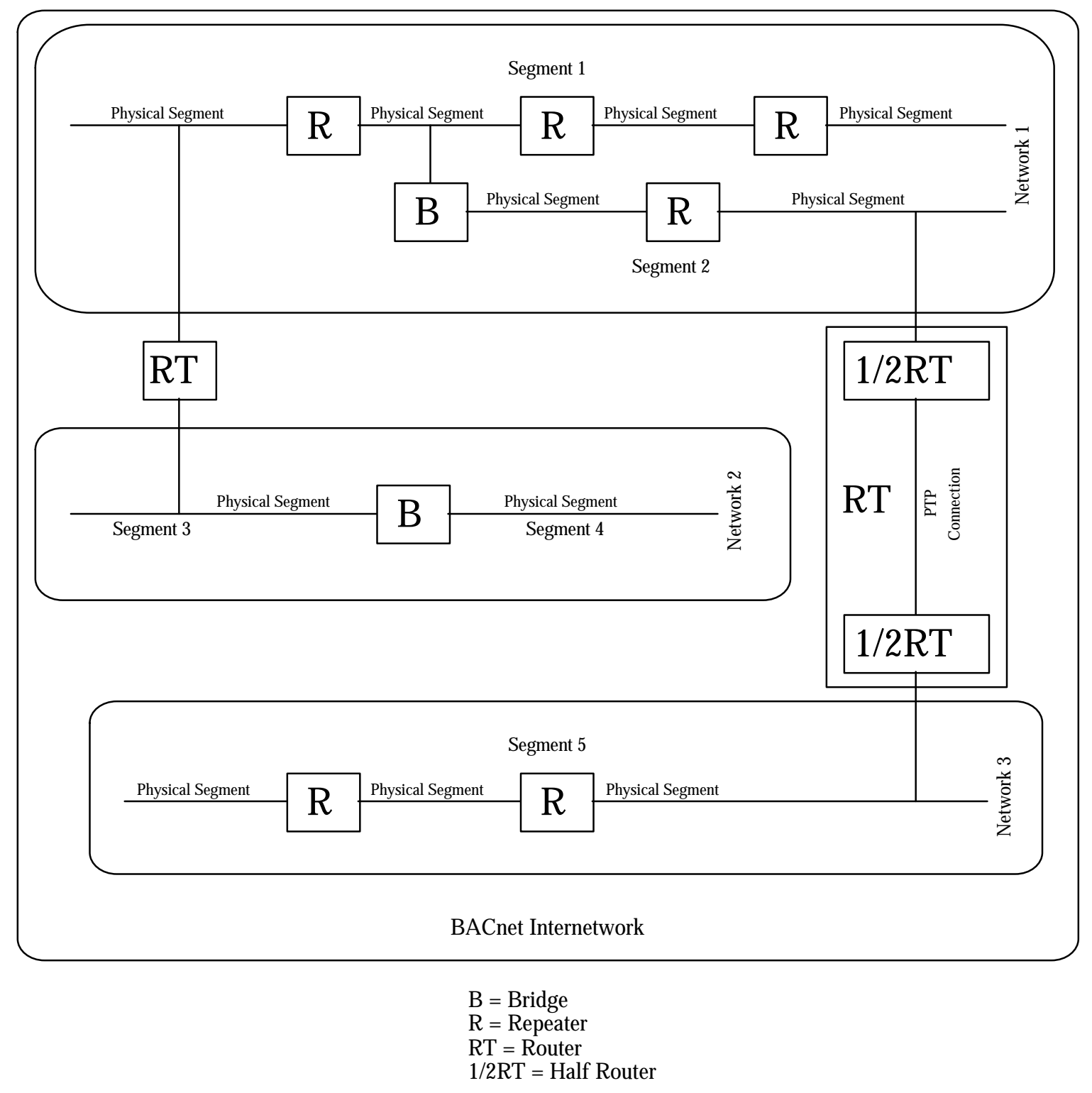

Figure 6-1. An Example of a BACnet Internetwork 
The simplest configuration is a network comprised of a single physical segment with all devices directly attached to it. The issue is then which local area network (LAN) type to select. This is discussed in 6.2. Since all LANs have some distance limitation, multiple physical segments may have to be joined by repeaters or bridges which selectively forward traffic based on knowledge of where specific devices are located. If several networks need to be interconnected, BACnet routers are available that can join networks using the same, or different, LAN technology. A key point is that slower networks should not be used to interconnect faster ones. Also, BACnet should be used throughout unless there are existing "legacy" networks to which a connection must be made. Such connections require the use of translators or "gateways" which almost inevitably affect performance, throughput, and functionality in a negative way. See 6.8.

- Specify that BACnet shall be used throughout the building automation and control internetwork at all levels.

- Specify that the internetwork shall be configured such that, if three or more networks are involved with different performance characteristics, the faster networks shall be used to interconnect the slower ones.

\subsection{Local Area Network Selection}

BACnet provides a great deal of flexibility in configuring different LAN technologies for optimal price vs. performance tradeoffs to meet the particular needs of a facility. The ability to use multiple LAN technologies also permits BACnet systems to accommodate new network technologies in the future while maintaining backward compatibility with installed system components.

BACnet currently supports four different LAN technologies, each one having particular pros and cons. Figure 6-2 illustrates the range of options presently in BACnet. Each technology is shown as a bubble. This reflects the fact that even within one LAN technology there are choices of media, topology, and in some cases speed that effect the price and performance.

Sections 6.2.1 - 6.2.5 explain in more detail the important features that should be considered when deciding on the appropriate LAN technologies to use. 


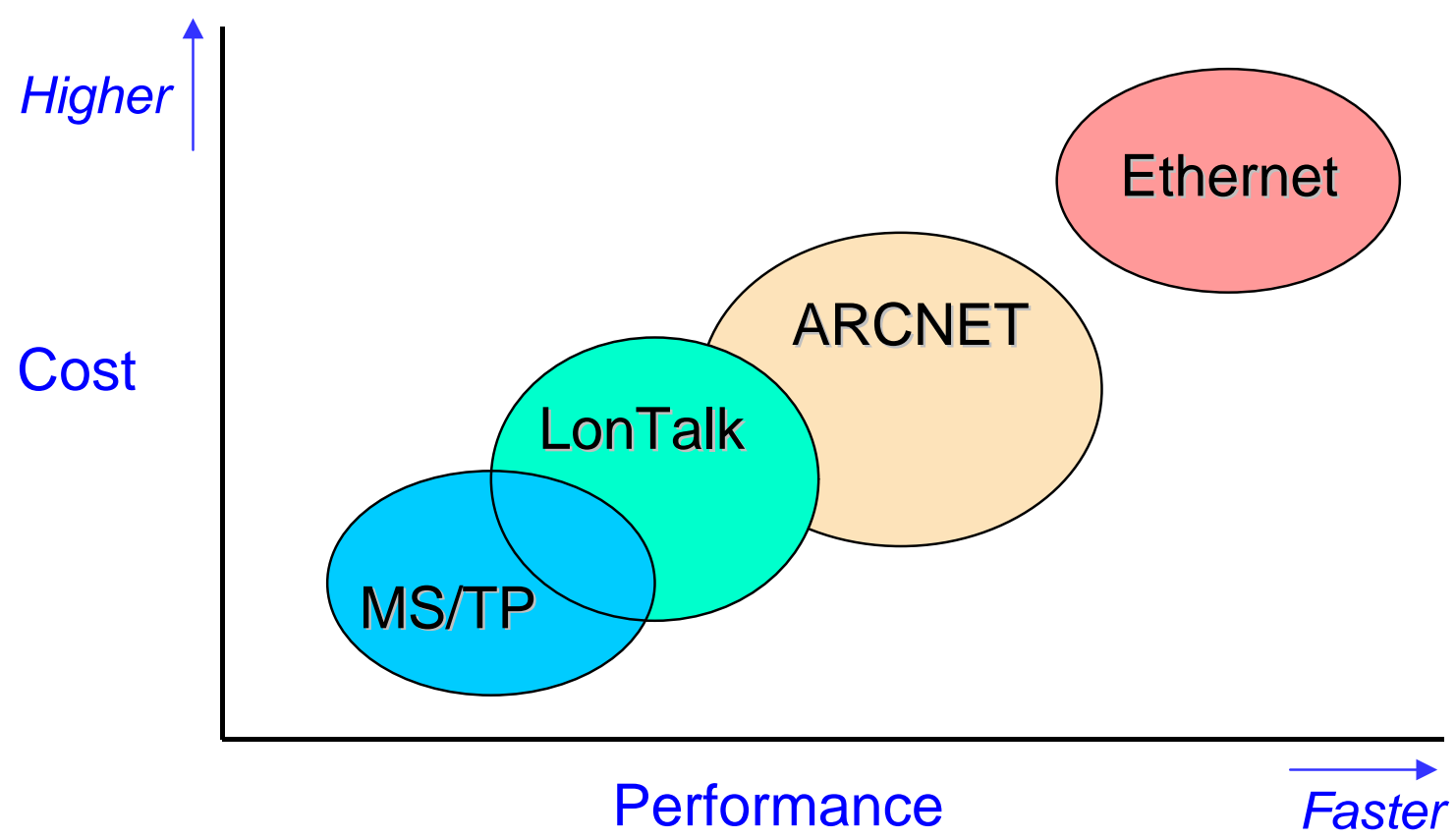

Figure 6-2. Cost vs. Performance for BACnet LAN Options

\subsubsection{ISO 8802-3, Ethernet}

ISO 8802-3 is commonly known by the name "Ethernet." This is probably the most widely used LAN technology in the world today, primarily because of its ubiquitous presence in office and business networks. It is the fastest LAN technology used in BACnet. Most building control companies offer Ethernet as an option for connecting high-end controllers with each other and with workstations. The key features of Ethernet are summarized in Table 6-1.

Table 6-1. Features of ISO-8802-3, Ethernet

\begin{tabular}{|l|l|}
\hline Pros & Cons \\
\hline$\bullet$ international standard & $\bullet$ relatively high cost \\
- already used in most buildings & $\bullet$ distance limitations \\
$\bullet$ variety of media $($ UTP, coax, fiber optic) & $\bullet$ non-deterministic \\
- very fast $(10$ Mbps or $100 \mathrm{Mbps})$ & \\
- easy to interface with PCs & \\
- the protocol is implemented in a chip & \\
- no special development tools & \\
\hline
\end{tabular}

Each of the media options has different cost, different length limitations, and different susceptibility to electrical interference. Coaxial cables are the lowest cost and they are restricted to a bus topology. Unshielded twisted pair (UTP) wiring is done in a star configuration and requires the use of hubs. This is a very robust architecture and offers maximum flexibility with respect to the physical location of the connected devices but it costs more because of the hubs. Fiber optic cables have the most immunity to electrical interference, can span longer distances, and are the most expensive option. It is possible to mix the various media in a single system. 
The 10 Mbps Ethernet technology is sufficient to meet the needs of most building control applications. Under some circumstances it may be desirable to use an existing $100 \mathrm{Mbps}$ LAN for a portion of the control system, a workstation for example. It is possible to connect $10 \mathrm{Mbps}$ segments to 100 Mbps segments by using special hubs.

Although Ethernet is the fastest LAN option in BACnet it is non-deterministic. This means that it is not possible to guarantee that a device will be able to transmit a message within a specific amount of time. This is a consequence of the fact that Ethernet uses a contention-based scheme to regulate access to the transmission medium. A device can transmit a message anytime the network is quiet. If two devices decide to transmit at the same time a collision occurs. Collisions only become a problem in networks with high traffic volumes. If the network is properly designed and maintained this difficulty can be avoided. In this context, "proper design" may involve, among other things, distributing nodes likely to generate high traffic volume onto more than one Ethernet segment and using bridges between segments that isolate "local" traffic so that it is not propagated to all nodes.

Specifying Ethernet LANs

Because of its cost, an Ethernet LAN is generally used as a backbone network connecting workstations and supervisory controllers together. The various options available make it necessary to indicate which ones are acceptable.

- Specify which devices in the control system should reside on the Ethernet LAN.

- Specify the transmission medium option that is to be used. If more than one will be used, indicate which one is to be used where, and the devices that are to be connected. If hubs are needed, specify the number of ports and the kind of medium for each port.

- Specify whether $10 \mathrm{Mbps}$ Ethernet or $100 \mathrm{Mbps}$ Ethernet is to be used.

\subsubsection{ANSI/ATA 878.1, ARCNET}

ARCNET (ANSI/ATA 878.1) is a LAN technology that is slower than Ethernet but it is deterministic, meaning that it is possible to determine the maximum delay before a device will be able to transmit a message. ARCNET has been popular in some building control product lines as a relatively high-speed network for connecting high-end controllers together and with workstations. Ethernet has gradually been diminishing ARCNET's popularity for this application.

The newest generation of ARCNET chips has a scaleable speed and can be used with EIA-485 signaling. This is becoming a popular option for use with unitary controllers. The key features of ARCNET are summarized in Table 6-2. 
Table 6-1. Features of ARCNET

\begin{tabular}{|l|l|}
\hline Pros & Cons \\
\hline - ANSI standard & $\bullet$ single source chip \\
- deterministic response & $\bullet$ distance limitations \\
- variety of media (UTP, coax, fiber optic) & $\bullet$ too costly for low-end controllers \\
- fast (150Kbps - 7.5Mbps) & \\
- high performance for medium cost & \\
- the protocol is implemented in a chip & \\
- no special development tools & \\
\hline
\end{tabular}

Specifying ARCNET LANs

If ARCNET is being used as a LAN for unitary controller networks there may be several of them in the control system. The specifications need to be clear for each one.

- Specify which devices in the control system should reside on the ARCNET LAN.

- Specify the transmission medium option that is to be used. If more than one will be used, indicate which one is to be used where and the devices that are to be connected. If hubs are needed, specify the number of ports and the kind of medium for each port.

- Specify how ARCNET addresses are to be assigned (see 6.3.1).

\subsubsection{Master-Slave/Token-Passing, MS/TP}

The Master-Slave/Token Passing protocol (MS/TP) was created by ASHRAE to meet the needs of low-cost building controllers and is unique to BACnet. The MS/TP protocol is implemented using EIA-485 signaling. MS/TP can be used in a master-slave mode, a peer-to-peer token passing mode, or a combination of the two. As a practical matter the speed is limited to about 76 Kbps. Higher speeds are theoretically possible but they would require a dedicated chip for reliable operation. The added complexity and cost would make LonTalk or ARCNET better choices at higher speeds.

MS/TP is the lowest cost LAN option in BACnet. It is designed for implementation on standard single-chip microprocessors without the addition of outboard hardware for timing and transceiver interfaces. The key features of MS/TP are summarized in Table 6-3. 
Table 6-3. Features of MS/TP

\begin{tabular}{|l|l|}
\hline Pros & Cons \\
\hline$\bullet$ ANSI standard & $\bullet$ single media \\
$\bullet$ low cost & $\bullet$ limited speed (9.6Kbps - 76Kbps) \\
$\bullet$ & \\
can be implemented in single chip & \\
$\bullet$ deterministic response & \\
$\bullet$ no special development tools & \\
\hline
\end{tabular}

Specifying MS/TP LANs

MS/TP LANs will generally be used for unitary controller networks so there may be several of them in the control system. The specifications need to be clear for each one.

- Specify which devices in the control system should reside on the MS/TP LAN.

- Specify the baud rate to be used.

- Specify how MS/TP addresses are to be assigned and if slave devices will be used how the address space is to be divided between slaves and masters (see 6.3.2).

\subsubsection{EIA-709.1, LonTalk}

LonTalk is a technology developed by the Echelon Corporation ${ }^{2}$. It has recently been adopted as an EIA standard for home automation networks. Like Ethernet and ARCNET, LonTalk is implemented in a special chip. LonTalk provides the most flexibility in terms of media options. Transceivers are available for traditional wired LANs (UTP, coax, fiber optic) as well as wireless communication (radio frequency (RF) and infrared (IR)) .

LonTalk allows scaleable speeds up to $1.25 \mathrm{Mbps}$. It is comparable to MS/TP at the low end, though the hardware is more costly in most cases, and ARCNET at the higher end (above 150 $\mathrm{Kbps})$. As the speed scales upward from $150 \mathrm{Kbps}$, ARCNET is somewhat more attractive in terms of hardware cost.

LonTalk has the unique distinction of being the only BACnet LAN technology that requires special development tools. These tools are only available from Echelon Corporation.

The LonTalk protocol is often confused with LonMark. They are totally different things. Devices using the LonTalk protocol do not interoperate unless some additional constraints are added that are specific to the intended application of the device. The BACnet application layer protocol

\footnotetext{
${ }^{2}$ Certain trade names and company products are mentioned in the text or identified in an illustration in order to adequately specify the equipment used. In no case does such an identification imply recommendation or endorsement by the National Institute of Standards and Technology, nor does it imply that the products are necessarily the best available for the purpose.
} 
defines these constraints. In BACnet, LonTalk is no different than any other LAN technology in that it is simply used to convey BACnet messages between devices.

LonMark is the name given to a set of implementer's agreements that make use of application features of the LonTalk protocol to achieve the same goal of interoperability. LonMark products are not compatible with BACnet. In order to mix BACnet devices and LonMark devices in the same control system, a gateway is required. It is no different than combining BACnet devices with devices using any proprietary communication protocol. Gateway issues are discussed in section 6.8. The key features of LonTalk are summarized in Table 6-4.

Table 6-4. Features of LonTalk

\begin{tabular}{|l|l|}
\hline Pros & Cons \\
\hline $\begin{array}{l}\bullet \text { variety of media (UTP, coax, RF, IR, } \\
\text { fiberoptic) }\end{array}$ & $\bullet$ non-deterministic \\
$\begin{array}{l}\text { - scaleable speed (32K to } 1.25 \mathrm{Mbps}) \\
\bullet \text { variety of media (UTP, coax, fiber optic) }\end{array}$ & $\begin{array}{l}\bullet \text { distance limitations } \\
\text { - special development tools required } \\
\end{array}$ \\
\hline
\end{tabular}

Specifying LonTalk LANs

LonTalk LANs will generally be used for unitary controller networks so there may be several of them in the control system. The specifications need to be clear for each one.

- Specify which devices in the control system should reside on the LonTalk LAN.

- Specify the media, speed and topology to be used.

- Specify how LonTalk addresses are to be assigned (see 6.3.3).

\subsubsection{Point-to-Point, PTP}

The BACnet Point-to-Point protocol offers a way to interconnect individual devices or networks using serial asynchronous connections such as provided by dial-up modem-to-modem links. The devices on either end of the PTP connection act as "half-routers" and, once the connection is established, appear as routers to devices on their respective networks. See Figure 6-1. If it is desired to remotely access a BACnet network via a dial-up connection, PTP should be specified.

- Specify that PTP shall be used as the means to attach to a BACnet LAN via a dial-up connection. Also specify that the optional password protection shall be provided.

\subsection{Specifying MAC Addresses in a Consistent Manner}

Every device in a BACnet system has a media access control (MAC) address. The MAC address is combined with the network number to uniquely identify each device for the purpose of delivering a message. This is similar to the way a street address is combined with a city and state to address a letter. For Ethernet networks a unique MAC address is assigned when the communication chip is manufactured. No special address configuration is necessary for BACnet 
devices using Ethernet. For other BACnet LANs, MAC addresses must be configured specifically for each installation.

In a multi-vendor environment it is important to manage the assignment of these addresses to ensure that there are no duplicates on the same network. There is no problem with duplicate MAC addresses on different networks, just like there is no problem with having more than one 123 Main Street as long as they are in different cities. A site-specific plan for assigning MAC addresses should be developed and vendors should be required to follow it. Guidance on how to do this for each of the relevant BACnet LANs is given below. It is also important to document the assigned addresses so that future changes to the system do not cause conflicts.

- Specify that MAC addresses shall be assigned as designated by the owner.

- Specify that a list of all assigned MAC addresses be included in the system documentation.

\subsubsection{Ethernet MAC Addresses}

For Ethernet networks a unique MAC address is assigned when the communication chip is manufactured. No special address configuration is necessary for BACnet devices using Ethernet.

\subsubsection{ARCNET MAC addresses}

The valid MAC addresses for BACnet devices using ARCNET are 1-255 (0 is reserved for broadcasts). Thus there can be at most 255 ARCNET devices on the same network. If the ARCNET LAN is part of an internetwork, meaning that there are two or more networks in the system, then there must be one or more routers. It is a good convention to reserve particular addresses to be used for ARCNET routers. If the ARCNET LAN is directly connected to only one other network then a single address is sufficient. If the ARCNET LAN is a backbone network then a range of addresses will be needed. Using the same router addresses on every ARCNET LAN in the system makes it easier to identify routers when troubleshooting problems. It may also make it easier to program controllers that need to know the router's address.

- The site addressing plan should reserve an address or a range of addresses to be used by ARCNET routers. For the case where one router per ARCNET network is sufficient, the value 255 is recommended. For the case where more than one router per network will be present, assigning addresses in decreasing order beginning with 255 is recommended.

If more than one vendor will be providing ARCNET devices that will reside on the same network or if that may be the case in the future, it is important to ensure that duplicate addresses are not accidentally assigned. One way to do this is to assign a range of addresses to each vendor. The vendors can select any address within the assigned range but cannot use addresses outside of their assigned range. All addresses used must be documented so that future changes can be managed.

- The site addressing plan should reserve a range of addresses for each vendor. It is recommended that ranges begin with the smallest address that is not in a previously assigned range. 


\subsubsection{MS/TP MAC addresses}

The valid MAC addresses for BACnet devices using MS/TP are 0-254 (255 is reserved for broadcasts). Thus there can be at most $255 \mathrm{MS} / \mathrm{TP}$ devices on the same network. There is an additional constraint because the address space is divided between master devices and slave devices. Addresses 128-254 are reserved for slaves. Addresses 0-127 are valid for both masters and slaves. The portion of the address space that is actually used for masters in a particular installation is determined by the value of the Max_Master property of the Device object.

The MS/TP LAN was designed for connecting low-cost controllers typically used for unitary or application specific controllers. It should not be used as a backbone LAN so that there should be at most one router to other LANs in the system. A router must be a master. It is recommended that MAC address 0 be reserved for use by the MS/TP router.

- The site addressing plan should reserve the address 0 for use with MS/TP routers.

If more than one vendor will be providing MS/TP devices that will reside on the same network or if that may be the case in the future, it is important to ensure that duplicate address are not accidentally assigned. One way to do this is to assign a range of addresses to each vendor. This applies to both master addresses and slave addresses. The vendor can select any address within the assigned range but cannot use addresses outside of their assigned range. All addresses used must be documented so that future changes can be managed.

- The site addressing plan should reserve a range of master addresses and slave addresses for each vendor as appropriate. It is recommended that ranges begin with the smallest address that is not in a previously assigned range.

The address space in the range 0 - 127 should be apportioned between masters and slaves according to the needs of the system. If Max_Master is writable using BACnet services, there is an advantage to assigning the smaller addresses first and configuring Max_Master to the highest address actually used instead of using the default value of 127 . This it true even if none of the address space will be used for slave devices. This approach will reduce the amount of time used to search for new stations that have entered the network and increase the bandwidth available for normal communication. If more master devices are added to the network at a later time it would be necessary to update the value of Max_Master in each of the master devices.

\subsubsection{LonTalk MAC addresses}

Like Ethernet, LonTalk neuron chips are manufactured with a unique address or Neuron_ID. Other addressing schemes can also be used that are based on (domain, subnet, node) configurations or (domain, group, member) configurations. If the Neuron_ID is to be used as the sole means of addressing for these devices no additional configuration is necessary. If one of the other addressing schemes is to be used then the owner needs to create and impose constraints to ensure that addresses are not duplicated. 


\subsection{Network Numbering Conventions}

A BACnet control system can contain up to 65,535 interconnected networks. This provides great flexibility for integrating the control systems in separate buildings. The ability to interconnect buildings provides opportunities for aggregating and managing utility loads in a deregulated utility environment, remote maintenance support, and centralized collection of energy performance data.

BACnet requires that each network in a system be assigned a unique network number. This means that if separate buildings are to be connected the assignment of network numbers must be managed so that no duplicate numbers occur. A GSA-wide plan for assigning network numbers should be developed and vendors should be required to follow it.

- Specify that network numbers shall be assigned as designated by the owner.

The recommended approach for assigning network numbers is to allow each region to use the entire network number domain within that region. The region would assign a range of numbers to each building. The people directly responsible for the building would subdivide this range in a manner appropriate to the building. For example, the division could be by network technology, by floor, or by wing. An example may make this idea clearer.

Consider a region where there are no more than 655 buildings. The network number domain could be divided as follows:

$$
\begin{aligned}
& \text { BBBFF } \\
& \text { where: } \begin{aligned}
\mathrm{BBB}= & \text { a number between } 1 \text { and } 655 \text { assigned to each building } \\
\mathrm{FF} & =00 \text { for the building backbone network } \\
\mathrm{FF} & =1-35 \text { indicating the floors or separate systems in the } \\
& \text { building }
\end{aligned}
\end{aligned}
$$

Note that the network numbers in the range where $\mathrm{BBB}=000$ are not assigned. These network numbers might be reserved for special purposes, such as temporary dial-up connections.

If a region has more than 655 buildings or has buildings with more than 35 floors then it might be necessary to sub-divide the region and use an entire network number domain for each subregion.

The consequence of these separate domains is that it will not be possible to integrate buildings that are in separate domains without using some additional mechanism to make the network numbers unique. This could be done if the central management site uses BACnet/IP to connect to each domain. By assigning each domain to a separate IP port it would become possible to distinguish otherwise identical network numbers. This would allow a central facility to communicate with all of the buildings but each building would only be able to communicate directly with devices that are within the same domain.

GSA already maintains a nation-wide database of buildings that includes a numbering system to uniquely identify each building. This numbering system has a range that is too large be used directly for assigning BACnet network numbers. For each region or sub-region that has a 
separate BACnet network domain, a mapping should be created to link the GSA building number with the range of BACnet network numbers that are assigned to that building.

It is important to note that any network number management approach that guarantees each network has a unique number meets the requirements of BACnet.

\subsection{Device Object Identifier Conventions}

A BACnet control system can contain up to 4,194,305 devices. This constraint comes from the fact that each device must have a unique value for the Object_Identifier property of the Device object. This uniqueness is the basis for BACnet services that permit dynamic location of information and binding of addresses (the process of determining how to address a message in order to communicate with a particular device).

In a multi-vendor and/or multi-building environment the assignment of the Device Object_Identifier value for each device must be managed so that no duplicate numbers occur. A GSA-wide plan for assigning Device Object_Identifier values should be developed and vendors should be required to follow it.

- Specify that Device Object_Identifier properties shall be assigned values as designated by the owner.

The recommended approach for assigning Device Object_Identifier values is to make use of the unique building numbers used to manage network numbers (the BBB field in the example above). In a given building the object identifier would end with the assigned building number. The remainder of the identifier number space would be divided in a manner appropriate to the building. An example of how this might work is as follows:

\section{XXFFBBB}

where: $\mathrm{XX}=$ a number in the range $00-40$

$\mathrm{FF}=00$ for the building backbone network

$\mathrm{FF}=1-35$ indicating the floors or separate systems in the building

$\mathrm{BBB}=$ a number between 1 and 655 assigned to each building

For ARCNET and MS/TP networks the XX field could also be used to assign the MAC address. See 6.3.

This management approach has the advantage of providing a great deal of information to someone troubleshooting communication problems because the physical location of the device can be deduced from the Object_Identifier property of the Device object. It also makes many valid Object_Identifier values unusable because they don't fit into the template. In this example, at most 41 devices could be present on each network for a total of 1,476 in the entire building. If the building does not use, and will never use all 36 networks, then the number space for unused networks can be added to existing networks to get around the limitation of 41 devices per network. 
This would work fine for many buildings but may be too restrictive for very large buildings. If the $\mathrm{XX}$ and FF fields were combined into one range that was assigned serially as devices were installed, the total number of possible BACnet devices increases to 4,195 and they could be arbitrarily assigned to different networks. These kinds of decisions can be made on a buildingby-building basis. The critical factor is that the range of possible values be restricted to a set that will never conflict with assignments made in other buildings in the same network domain.

\subsection{Use of BACnet with the Internet Protocols}

BACnet provides two distinct ways that messages can be conveyed across internets that use IP, the Internet Protocol. By means of these techniques, wide area BACnet internetworks can be created that span the country or the globe.

\subsubsection{Annex H Tunneling Routers}

BACnet Tunneling Routers (BTR) are devices that appear as traditional routers to the devices that are on the same LAN as the BTR but use IP to communicate with peer BTRs on distant LANs. This is accomplished by configuring routing tables in each BTR that consist of (BACnet Network Number, IP Address) pairs. The mechanism is illustrated in Figure 6-3.

The use of BTRs is relatively inexpensive and has the advantage that the individual BACnet devices need not themselves be "IP literate." In other words, BTRs can be used with existing BACnet networks that also have a connection to an IP internet, intranet, or the Internet with a capital "I". 


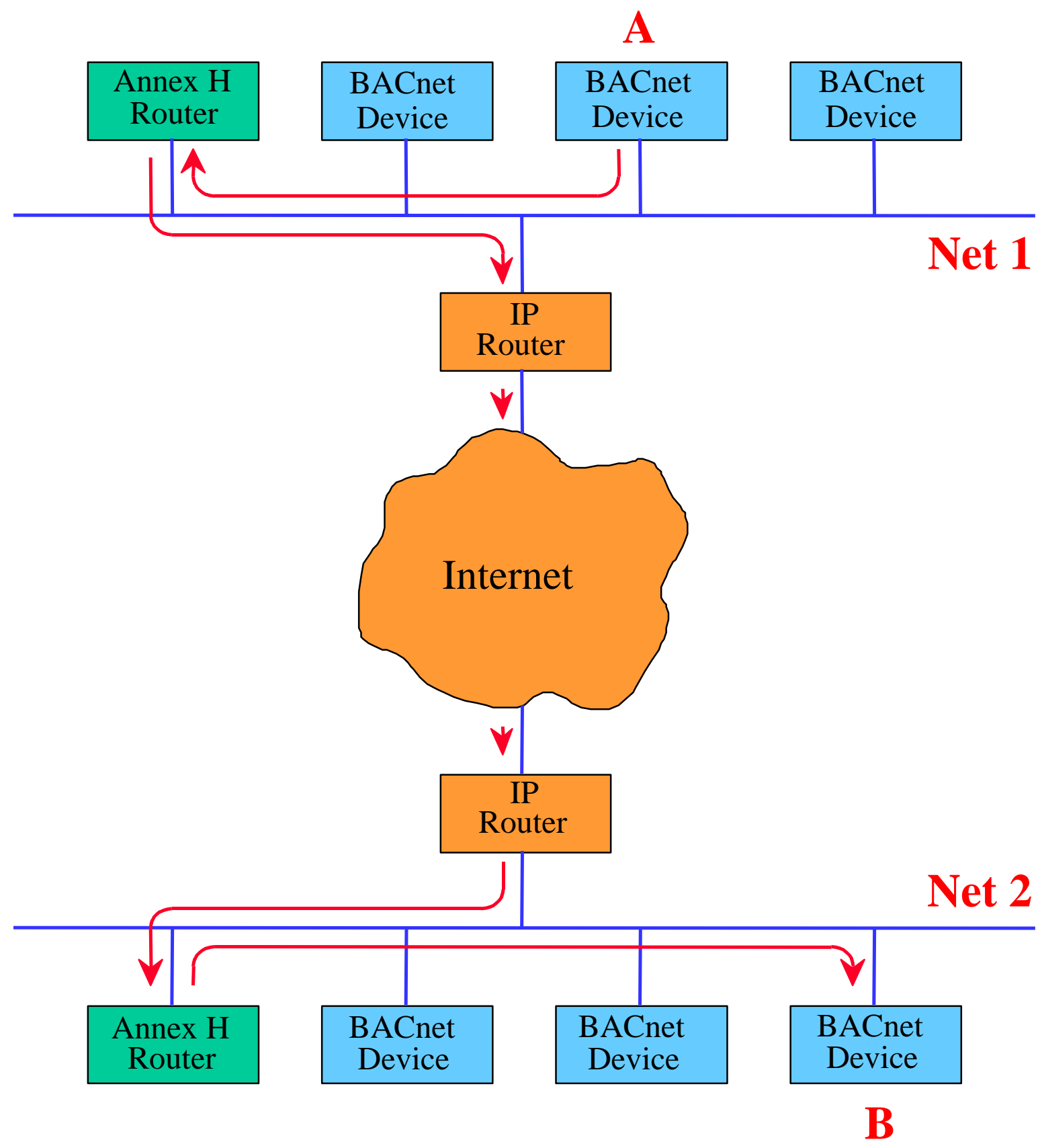

Figure 6-3. An IP Connection using Annex H Tunneling Routers

\subsubsection{Addendum 135a, BACnet/IP}

In the case of BACnet/IP, the addendum to BACnet adopted in January 1999, the individual BACnet devices themselves are IP-capable. Thus no tunneling routers are needed and the devices can communicate among themselves directly. A problem arises, however, when it is necessary to convey broadcast messages from one IP subnet to another since most IP routers do not pass such messages. To get around this, BACnet/IP specifies the use of a device called a "BACnet Broadcast Management Device" (BBMD). A BBMD acts essentially like a BTR but only for 
broadcast messages. BACnet/IP also specifies how IP multicasting can be used to propagate broadcasts if the IP routers support it. Multicasting eliminates the need for BBMDs.

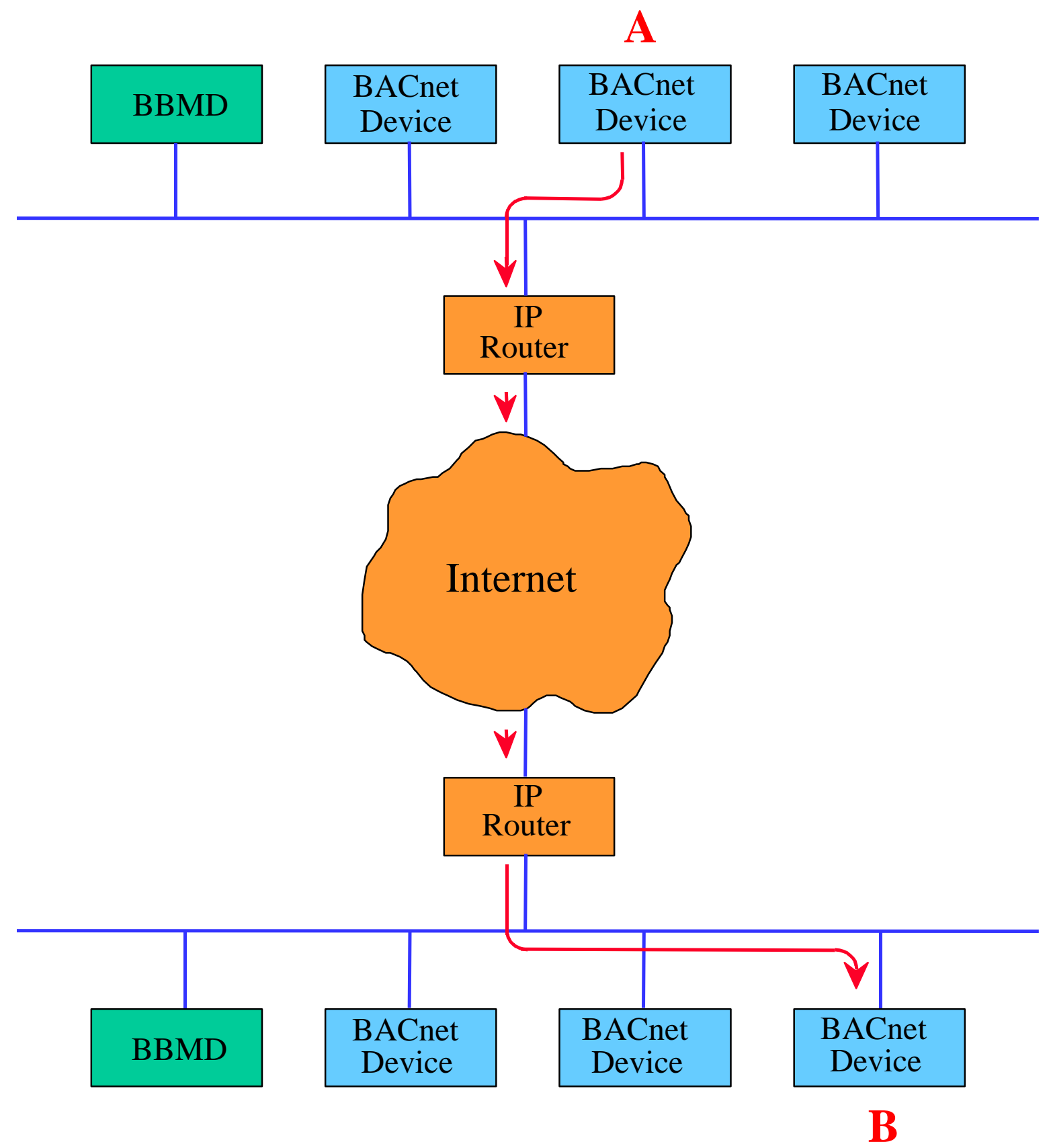

Figure 6-4. BACnet/IP Devices Communicate with Each Other Directly

Note in Figure 6-4 devices A and B are on the same BACnet network even though they are on different IP subnets. Thus multiple IP subnets can be combined to form a single BACnet network. BACnet/IP also allows "foreign devices," i.e., devices not on the same subnet with any of the other BACnet devices, to "join" the BACnet internet. This capability will prove useful for workstations that have internet access but are remote from the site where the BACnet devices are located. 
- Specify, if existing BACnet non-IP devices are to be interconnected via an IP network, that BACnet Tunneling Routers conforming to Annex H shall be provided.

- Specify, for new networks which need IP connectivity, that devices shall be provided that conform to Addendum 135a, BACnet/IP. For broadcast distribution, BBMDs shall also be provided or appropriate arrangements made for the use of IP multicasting.

\subsection{Routers - Interconnecting Multiple BACnet Networks}

Routers in BACnet are used for two distinct purposes. The first is to join together networks that use different LAN technologies, e.g., a BACnet Ethernet LAN to a BACnet MS/TP LAN. The second is to join together networks that use the same technology but have exhausted their supply of MAC addresses. An example of this would be a router that connects two ARCNET LANs, each of which can have at most only 255 devices. Several items should be specified.

- Specify that it shall be the contractor's responsibility to configure each router using the network numbering scheme agreed to for the project.

- Specify that each router shall be configured such that all network layer error messages shall be directed to a specific workstation using the BACnet ConfirmedTextMessage service.

- Specify that it shall be the contractor's responsibility to initially configure each router with routing tables containing all network numbers that are part of the project's internet.

- Specify that the router shall be able to receive messages at each port of any length that is valid for the LAN technology connected to that port, and to forward the message to any directly-connected network that can convey a message of that size.

\subsection{Message Segmentation}

Message traffic data collected from operating BACnet systems indicate that the most commonly used BACnet messages are small. However, it is sometimes desirable to be able to exchange large messages. For example, uploading and downloading a device's database and programs may require the exchange of considerable information.

A manufacturer may choose to restrict the message buffer size in a device as a way to optimize memory resources. Under these circumstances it may become necessary to support message segmentation in order to exchange large messages. LAN technologies also impose constraints on maximum message sizes. A consistent policy on message sizes and segmentation is needed to insure interoperability in multi-vendor systems.

- Specify that all BACnet devices shall support the reception of messages up to the largest size permitted by the LAN technology or support reception of segmented messages. 
- Specify that all BACnet devices shall support the transmission of messages up to the largest size permitted by the LAN technology or support transmission of segmented messages.

- Specify that all workstations shall support both segmented message transmission and segmented message reception.

- Specify that all BACnet Building Controllers (see B.2) shall support both segmented message transmission and segmented message reception.

\subsection{Gateways - Connecting to Legacy Systems}

In some retrofit projects it is not economically viable, or necessary, to replace the entire building control system at one time. Expansion or replacement of the system may take place in stages. Under these circumstances it is necessary to find a way to connect the remaining legacy controllers with the new BACnet controllers. This is accomplished by using a device called a "gateway." A gateway is a device that translates between BACnet and the non-BACnet communication protocol.

Computers require precise, rigidly defined rules or protocols for communication. Protocol translation, like language translation, is an imperfect art. Concepts that are easy to express in one language or protocol may be difficult or even impossible to translate into another. Gateways usually provide access to only a subset of the information that is available in the non-BACnet system. Complicated tasks such as scheduling and alarm processing may not be possible at all. For these reasons gateways should be avoided when possible and when they are necessary their capabilities and performance must be clearly specified.

Which devices can communicate with each other?

Gateways do not necessarily permit any arbitrary pair of devices to communicate with each other. For example, a workstation could be capable of connecting to a BACnet LAN and also to a non-BACnet LAN and provide the operator with access to information from both systems. This approach may not allow communication between the BACnet controllers and the non-BACnet controllers.

- Specify that the operator workstation shall display information from both the BACnet and non-BACnet devices. If appropriate, specify which other devices should also be able to communicate through the gateway.

Uni-directional or bi-directional communication?

Gateways may be uni-directional, meaning that a BACnet device can access information from a non-BACnet device but the non-BACnet devices cannot access information from BACnet devices. The reverse is also possible where a non-BACnet device can access information from BACnet devices but BACnet devices cannot access information from the non-BACnet devices. Gateways may also be bi-directional. 
- Specify which way information must flow through the gateway or that the gateway should provide bi-directional communication.

Which points are readable?

Gateways have a limited capacity. Not all information contained in the systems being interconnected is necessarily accessible through the gateway.

- Specify which non-BACnet points are to be readable from the BACnet side of the gateway and which BACnet objects are to be readable from the non-BACnet side of the gateway.

Which points are changeable?

The ability to view information through the gateway does not imply an ability to make changes or to command actions through the gateway.

- Specify which non-BACnet points are to be modifiable from the BACnet side of the gateway and which BACnet objects are to be modifiable from the nonBACnet side of the gateway.

Expansion

Control systems tend to change over time. A gateway needs to be able to accommodate foreseeable control system changes.

- Specify how much expansion capacity is required in the gateway.

Archiving controller programs and configuration databases

Many control systems provide a way to archive control programs and configuration databases by uploading the pertinent files from the controller. These archives can be used to restore the controller to a known configuration in the event of an equipment failure. Gateways may not be capable of transferring this kind of information.

- Specify how controller program and configuration database archives are to be created and maintained.

Trending

The collection of trend data is handled in many different ways in proprietary control systems. A gateway may permit a workstation to access the points that need to be trended but may not be able to transfer trend log files that are created by a controller. It is important to understand the trending capabilities of the legacy system and to ensure that the gateway has the necessary capability to support the trending needs of the application. For additional information about trending see 3.4. 
- Specify any gateway features that will be needed to support collection of trend data.

Scheduling

The methods used to schedule control system actions are handled in many different ways in proprietary control systems. If the scheduling needs of the facility require passing schedule information through the gateway, this capability must be specified. For more information about scheduling see 3.3.

- Specify any gateway features that will be needed to support scheduling.

Alarm and Event Handling

The detection, notification, and acknowledgment of alarms and other events are handled in many different ways in proprietary control systems. It is important to understand the alarm and event handling capabilities of the legacy system and to ensure that the gateway has the necessary capability to support any needed alarm and event processing in the integrated system.

- Specify any gateway features that will be needed to support the detection, notification, and acknowledgment of alarms and other significant events.

\section{Practical Considerations in the Construction of BACnet Systems}

Several key issues directly affect the successful implementation and operation of a BACnetbased building automation system. These issues can be classified according to the phases of any construction project - from procurement through construction and ongoing operations and maintenance. In the sections that follow, some general guidelines and recommendations are offered to assist the BAS project team in the procurement, construction, commissioning, and operation/maintenance phases of a BAS project.

\subsection{Procurement Phase}

A successful BAS implementation requires a careful vendor procurement process. The end product of a control system design should be a complete set of construction documents. The construction documents referred to here include the Request for Proposal (RFP) and the project's Technical Specifications.

The RFP document serves as the vendor's overview of the project, a set of instructions for bidding the project, and the specifying engineer's tool to evaluate competing BAS solutions. Therefore, there are several items that must be included in this document in order to ensure interoperability via BACnet, and to avoid misunderstandings and future implementation problems.

As previously stated, BACnet enables multi-vendor interoperability but in and of itself, does not require that multiple vendors participate in any given BAS project. If the project requires a multivendor environment, special attention should be made in the RFP to clearly define each scope of 
work along with its respective deliverables. In the process of this definition, the overall system design should be referenced to ensure that all system components (hardware and software) are accounted for within the individual scopes and to ensure that there is no overlap.

In order to ensure interoperability, the RFP should require the controls vendor to submit a description of the proposed system. This description should define the system architecture and explicitly identify "where and how" BACnet is to be implemented. At a minimum, the RFP should require that the description include the following items:

- Narrative providing an overview of the BAS system, primary components, workstation(s), and LAN architecture

- Identification of any clarifications or exceptions to the project specifications

- A listing and quantification of all microprocessor-based BAS equipment to be supplied and associated information "cut-sheets" for each

- A listing of references, as appropriate, for projects of a similar nature to the proposed BAS

- The option of requesting a formal BAS demonstration at either the vendor's local offices or customer location

In addition to the system description, a completed "Vendor Qualifications Form" should be developed. As part of this form, the vendor should be required to provide information on local office staffing, spare parts inventory, and the number of locally installed BACnet systems with references.

With the above information, the specifying engineer can ensure the vendor understands the requirements of the project, has sufficient experience, and has proposed a system that adheres to the Technical Specifications. If alternative approaches are provided in one or more exceptions to the specifications they must be carefully reviewed to determine whether or not they meet the project needs.

\subsection{Construction Phase}

After the controls vendor(s) are selected and the project is awarded, the submittals phase of construction begins. As part of the submittal review process, the control vendor(s) should be required by the Technical Specification to submit several key items to ensure interoperability. These items include a Protocol Implementation Conformance Statement (PICS) and detailed points lists. Coordination issues between the vendor(s) and Owner also require special consideration.

\subsubsection{Protocol Implementation Conformance Statements (PICS)}

As part of the vendor's submittal package, a PICS should be submitted for each BACnet device in the system. At a minimum, the PICS should demonstrate the device's BACnet capability by listing the following items: 
1. BACnet Standard Application Services Supported - This table confirms the BACnet services supported by the device. Refer to Annex A for detailed descriptions of these services.

2. Standard Object Types Supported - This table lists the device's supported object types as listed in Section 4.2. It also indicates if the object is dynamically creatable, dynamically deletable, optional supported properties, and writable properties.

3. Data Link Layer Options - Describes the network types supported for communications, e.g., Ethernet, ARCNET, or MS/TP.

4. Special Functionality - Describes any special exceptions the device may have to the BACnet protocol in order to perform any specific function.

5. Property Range Restrictions - Indicates, among other things, the number of characters allowed for the various text properties, such as Object_Name and Description.

The BACnet requirements of the Technical Specifications should act as the submittal review criteria. The information provided by the PICS should be compared to the Technical Specifications to ensure the device will function in the system as intended by the design.

\subsubsection{Points Lists}

The controls vendor(s) should also provide a detailed points lists for the system. In addition to standard I/O information, these points lists should contain the following items to ensure BACnet interoperability:

- Proposed I/O Names - An I/O point naming convention should be specified in the Technical Specifications in order to identify clearly system points. Verification of the vendor's proposed naming schemes should be performed.

- BACnet Object Description - This description includes the Object ID and Device ID for each I/O point. In a multi-vendor environment, special care should be taken to ensure that no device ID's are duplicated. Also, non-standard BACnet objects and properties should be documented including their structure and data types.

\subsubsection{Coordination}

Successful BACnet interoperability also depends on the interaction between vendors, in a multivendor environment, and between the vendor(s) and owner. To ensure this process takes place both effectively and proactively, the vendor should produce a coordination action-list that identifies all BACnet-related information requirements (e.g., each BAS vendor involved, HVAC equipment interfaces, owner LAN/WAN coordination, etc.), responsible parties, contact names, and critical dates. This action-list should be reviewed and updated at all BAS-related progress meetings. 


\subsection{Commissioning Phase}

During the commissioning phase, each control sequence should be verified along with the functionality of each workstation component including graphics, reports, trend logs, and so on. In order to verify the operation of the BACnet communications, insofar as it relates to functions above, it may prove useful to make use of a "protocol analyzer" to observe the message flow and content on the network. A protocol analyzer is a computer that can intercept messages on the communication medium and display their contents in an easy-to-understand format. Obviously knowledge of the BACnet protocol is a prerequisite to interpreting the captured network traffic. BACnet protocol analyzers are available from NIST in the form of "Visual Test Shell" software and also from at least one commercial vendor. The analyzer software can be installed on a portable laptop computer or on a system workstation, whichever is most convenient.

With a protocol analyzer an operator can verify that alarm messages are being directed to the proper recipients; that change-of-value notifications are being correctly produced; that time synchronizations are sent at the correct intervals; and so on. Also, if there is a particular problem that needs to be fixed, the protocol analyzer can be set to "trigger" its data capture based on a specific message, source device, destination device, time of day, or other defined condition. Likewise, the captured data can be filtered to eliminate messages that are not relevant to resolving the problem at hand. Experience has shown that most operational problems relate to improper device configuration or programming. In such cases, the use of a protocol analyzer can often pinpoint the cause of a problem within minutes.

When a project involves the expansion of an existing BACnet system, it is important to note that the vendor providing the new equipment may not be the same vendor that provided the operator workstation. It is important to have someone present during commissioning who understands the details of the workstation set-up and configuration.

\subsection{Operation and Maintenance Phase}

After commissioning and acceptance, ownership of the system is transferred from the controls vendor(s) to the owner. There are several Operation and Maintenance $(\mathrm{O} \& \mathrm{M})$ issues that must be addressed before this transfer occurs and as an on-going process throughout the life of the system that will effect future expansion and interoperability of the system.

\subsubsection{Record Documentation}

Before system acceptance occurs (and release of final retainage), the owner should be in possession of a complete set of record documentation including the following:

- "As-built" record drawings - Schematic drawings that represent the final system architecture, configuration, and input/output (I/O) points.

- I/O Points List - The I/O listings should include the name/description, display units, alarm limit(s)/definition, and BACnet object description, including Object ID and Device ID, for each $\mathrm{I} / \mathrm{O}$ point. 
- Non-Standard BACnet Objects - Documentation for any non-standard BACnet objects, properties, or enumerations utilized detailing their structure, data types, and any associated lists or enumerated values.

- Program records - Complete program descriptions of all control and application software per the system installation. This should include English narratives of control/application programs, flowcharts and actual source code/files.

As hardware and/or software modifications are made to the system, these records must be updated to reflect the current operating conditions of the system. As future expansion needs arise, the owner will be able to competitively bid out the work to controls vendors and provide them with the record documentation in order to ensure that the expansion will operate with the existing system.

\subsubsection{Operator Training}

Operator training becomes an integral part of BACnet interoperability when considering the expansion of an existing BACnet system. This process begins at the design phase when training requirements are specified in the Technical Specifications. The specifying engineer should include the following items in order to ensure that the operating personnel receive complete system training and are fully capable of operating and maintaining the system.

- Minimum Training Hours - Specify the minimum number of hours required by the controls vendor(s) for training of operator personnel. This should include on-site (hand-on) training as well as off-site (factory) training.

- Course Content - Specify the minimum course content requirements. In addition to basic system training, the content should also include an overview of the BACnet protocol and system network. Also included in the content should be skills to enable the operator to add, delete, and/or modify I/O points and create or edit system tabular reports and graphic displays at the Operator Workstation (OWS).

The owner and/or a representative should attend the training sessions to ensure that these training requirements are fulfilled. Such training could potentially decrease the owner's future system expansion costs by limiting the controls vendor(s) to only installing and commissioning field devices while the owner's operating personnel add and/or modify the system reports and graphics at the OWS and map the desired newly installed I/O points as provided by the controls vendor(s). 


\section{Annex A. BACnet Interoperability Building Blocks (BIBBs)}

BACnet Interoperability Building Blocks (BIBBs) are collections of one or more BACnet services. These are prescribed in terms of an "A" and a "B" device. Both of these devices are nodes on a BACnet inter-network. In most cases the "A" device will act as the user of data and the " $\mathrm{B}$ " device will be the provider of this data. In addition, certain BIBBs may also be predicated on the support of certain, otherwise optional, BACnet objects or properties and may place constraints on the allowable values of specific properties or service parameters.

\section{A.1 Data Sharing BIBBs}

These BIBBs prescribe the BACnet capabilities required to interoperably perform the data sharing functions enumerated in 3.1 for the BACnet devices defined therein.

\section{A.1.1 BIBB - Data Sharing-ReadProperty-A (DS-RP-A)}

The A device is a user of data from device B.

\begin{tabular}{|c|c|c|}
\hline BACnet Service & Initiate & Execute \\
\hline ReadProperty & $\mathrm{x}$ & \\
\hline
\end{tabular}

\section{A.1.2 BIBB - Data Sharing-ReadProperty-B (DS-RP-B)}

The $\mathrm{B}$ device is a provider of data to device A.

\begin{tabular}{|c|c|c|}
\hline BACnet Service & Initiate & Execute \\
\hline ReadProperty & & $\mathrm{x}$ \\
\hline
\end{tabular}

\section{A.1.3 BIBB - Data Sharing-ReadPropertyMultiple-A (DS-RPM-A)}

The A device is a user of data from device B and requests multiple values at one time.

\begin{tabular}{|c|c|c|}
\hline BACnet Service & Initiate & Execute \\
\hline ReadPropertyMultiple & $\mathrm{x}$ & \\
\hline
\end{tabular}

\section{A.1.4 BIBB - Data Sharing-ReadPropertyMultiple-B (DS-RPM-B)}

The B device is a provider of data to device A and returns multiple values at one time.

\begin{tabular}{|c|c|c|}
\hline \multicolumn{1}{|c|}{ BACnet Service } & Initiate & Execute \\
\hline ReadPropertyMultiple & & $\mathrm{x}$ \\
\hline
\end{tabular}




\section{A.1.5 BIBB - Data Sharing-ReadPropertyConditional-A (DS-RPC-A)}

The A device is a user of data from device B and requests that values be returned based upon specific criteria that are contained in the message.

\begin{tabular}{|c|c|c|}
\hline BACnet Service & Initiate & Execute \\
\hline ReadPropertyConditional & $\mathrm{x}$ & \\
\hline
\end{tabular}

\section{A.1.6 BIBB - Data Sharing-ReadPropertyConditional-B (DS-RPC-B) )}

The B device is a provider of data to device A based, conditionally, upon the selection criteria in the request from device $\mathrm{A}$.

\begin{tabular}{|c|c|c|}
\hline BACnet Service & Initiate & Execute \\
\hline ReadPropertyConditional & & $\mathrm{x}$ \\
\hline
\end{tabular}

\section{A.1.7 BIBB - Data Sharing-WriteProperty-A (DS-WP-A))}

The A device sets a value in device B.

\begin{tabular}{|l|c|c|}
\hline \multicolumn{1}{|c|}{ BACnet Service } & Initiate & Execute \\
\hline WriteProperty & $\mathrm{x}$ & \\
\hline
\end{tabular}

\section{A.1.8 BIBB - Data Sharing-WriteProperty-B (DS-WP-B)}

The B device allows a value to be changed by device $\mathrm{A}$.

\begin{tabular}{|l|c|c|}
\hline BACnet Service & Initiate & Execute \\
\hline WriteProperty & & $\mathrm{x}$ \\
\hline
\end{tabular}

\section{A.1.9 BIBB - Data Sharing-WritePropertyMultiple-A (DS-WPM-A)}

The A device sets multiple values in device B at one time.

\begin{tabular}{|l|c|c|}
\hline BACnet Service & Initiate & Execute \\
\hline WritePropertyMultiple & $\mathrm{x}$ & \\
\hline
\end{tabular}




\section{A.1.10 BIBB - Data Sharing-WritePropertyMultiple-B (DS-WPM-B)}

The $\mathrm{B}$ device allows multiple values to be changed by device $\mathrm{A}$ at one time.

\begin{tabular}{|l|c|c|}
\hline \multicolumn{1}{|c|}{ BACnet Service } & Initiate & Execute \\
\hline WritePropertyMultiple & & $\mathrm{x}$ \\
\hline
\end{tabular}

\section{A.1.11 BIBB - Data Sharing-COV-A (DS-COV-A)}

The $\mathrm{B}$ device is a provider of $\mathrm{COV}$ data to device $\mathrm{A}$.

\begin{tabular}{|l|c|c|}
\hline \multicolumn{1}{|c|}{ BACnet Service } & Initiate & Execute \\
\hline SubscribeCOV & & $\mathrm{x}$ \\
\hline ConfirmedCOVNotification & $\mathrm{x}$ & \\
\hline UnconfirmedCOVNotification & $\mathrm{x}$ & \\
\hline
\end{tabular}

Devices claiming conformance to DS-COV-B shall support a minimum of 5 simultaneous subscriptions.

\section{A.1.12 BIBB - Data Sharing-COV-B (DS-COV-B)}

The A device is a user of COV data from device B.

\begin{tabular}{|l|c|c|}
\hline \multicolumn{1}{|c|}{ BACnet Service } & Initiate & Execute \\
\hline SubscribeCOV & $\mathrm{x}$ & \\
\hline ConfirmedCOVNotification & & $\mathrm{x}$ \\
\hline UnconfirmedCOVNotification & & $\mathrm{x}$ \\
\hline
\end{tabular}

\section{A.1.13 BIBB - Data Sharing-COV-Unsubscribed-A (DS-COVU-A)}

The A device processes unsolicited COV data from device B.

\begin{tabular}{|c|c|c|}
\hline BACnet Service & Initiate & Execute \\
\hline UnconfirmedCOVNotification & & $\mathrm{x}$ \\
\hline
\end{tabular}

\section{A.1.14 BIBB - Data Sharing-COV-Unsubscribed-B (DS-COVU-B)}

The B device generates unsolicited COV notifications.

\begin{tabular}{|c|c|c|}
\hline BACnet Service & Initiate & Execute \\
\hline UnconfirmedCOVNotification & $\mathrm{x}$ & \\
\hline
\end{tabular}




\section{A.2 Alarm and Event Management BIBBs}

These BIBBs prescribe the BACnet capabilities required to interoperably perform the alarm and event management functions enumerated in 3.2 for the BACnet devices defined therein.

\section{A.2.1 BIBB - Alarm and Event-Notification-A (AE-N-A)}

The A device processes notifications about alarms and other events.

\begin{tabular}{|l|c|c|}
\hline \multicolumn{1}{|c|}{ BACnet Service } & Initiate & Execute \\
\hline ConfirmedEventNotification & & $\mathrm{x}$ \\
\hline UnconfirmedEventNotification & & $\mathrm{x}$ \\
\hline
\end{tabular}

\section{A.2.2 BIBB - Alarm and Event-Notification-B (AE-N-B)}

Device B generates notifications about alarms and other events.

\begin{tabular}{|l|c|c|}
\hline \multicolumn{1}{|c|}{ BACnet Service } & Initiate & Execute \\
\hline ConfirmedEventNotification & $\mathrm{x}$ & \\
\hline UnconfirmedEventNotification & $\mathrm{x}$ & \\
\hline
\end{tabular}

Devices claiming conformance to AE-N-B shall also support either Intrinsic or Algorithmic reporting.

\section{A.2.3 BIBB - Alarm and Event-ACK-A (AE-ACK-A)}

Device A acknowledges alarm/event notifications.

\begin{tabular}{|c|c|c|}
\hline BACnet Service & Initiate & Execute \\
\hline AcknowledgeAlarm & $\mathrm{x}$ & \\
\hline
\end{tabular}

\section{A.2.4 BIBB - Alarm and Event-ACK-B (AE-ACK-B)}

Device B processes acknowledgments of previously transmitted alarm/event notifications.

\begin{tabular}{|c|c|c|}
\hline BACnet Service & Initiate & Execute \\
\hline AcknowledgeAlarm & & $\mathrm{x}$ \\
\hline
\end{tabular}

In order to support this BIBB the device must also support acknowledgeable alarms. 


\section{A.2.5 BIBB - Alarm and Event-Summary-A (AE-ASUM-A)}

Device A requests summaries of alarms from device B.

\begin{tabular}{|c|c|c|}
\hline BACnet Service & Initiate & Execute \\
\hline GetAlarmSummary & $\mathrm{x}$ & \\
\hline
\end{tabular}

\section{A.2.6 BIBB - Alarm and Event-Summary-B (AE-ASUM-B)}

Device B provides summaries of alarms device A.

\begin{tabular}{|c|c|c|}
\hline BACnet Service & Initiate & Execute \\
\hline GetAlarmSummary & & $\mathrm{x}$ \\
\hline
\end{tabular}

\section{A.2.7 BIBB - Event-Summary-A (AE-ESUM-A)}

Device A requests event enrollments from device B.

\begin{tabular}{|c|c|c|}
\hline BACnet Service & Initiate & Execute \\
\hline GetEnrollmentSummary & $\mathrm{x}$ & \\
\hline
\end{tabular}

\section{A.2.8 BIBB - Event-Summary-B (AE-ESUM-B) )}

Device B provides event enrollments to device A.

\begin{tabular}{|c|c|c|}
\hline BACnet Service & Initiate & Execute \\
\hline GetEnrollmentSummary & & $\mathrm{x}$ \\
\hline
\end{tabular}

\section{A.3 Scheduling BIBBs}

These BIBBs prescribe the BACnet capabilities required to interoperably perform the scheduling functions enumerated in 3.3 for the BACnet devices defined therein.

\section{A.3.1 BIBB - Scheduling - A (SCHED-A)}

The A device manipulates schedules and calendars on the B device. The A device must support the DS-RP-A and DS-WP-A BIBBs. 


\section{A.3.2 BIBB - Scheduling - B (SCHED-B)}

The B device provides date and time scheduling of the values of specific properties of specific objects. In addition to supporting the DS-RP-B and DS-WP-B BIBBs, each device claiming conformance to SCHED-B must also possess at least one Calendar, one Schedule object, and one Command object. The Command object is required for scheduling actions in other devices.

The Schedule object must support at least 6 entries per day and at least one entry in the List_of_Object_Property_Reference property. The Schedule object must support a non-empty Exception_Schedule property.

The Command object must be capable of writing to objects in other devices. The Priority_For_Writing property in the Command object must be writable.

\section{A.4 Trending BIBBs}

These BIBBs prescribe the BACnet capabilities required to interoperably perform the trending functions enumerated in 3.4 for the BACnet devices defined therein.

\section{A.4.1 BIBB - Viewing and Modifying Trends - A (T-VMT-A)}

The A device displays trend data from the $\mathrm{B}$ device and manipulates trend log collection parameters in the B device.

\begin{tabular}{|c|c|c|}
\hline BACnet Service & Initiate & Execute \\
\hline ReadRange & $\mathrm{x}$ & \\
\hline
\end{tabular}

\section{A.4.2 BIBB - Viewing and Modifying Trends - B (T-VMT-B)}

The B device collects the trend log data records in an internal buffer. Each device claiming conformance to T-VMT-B must be able to support at least one Trend Log object.

\begin{tabular}{|c|c|c|}
\hline BACnet Service & Initiate & Execute \\
\hline ReadRange & & $\mathrm{x}$ \\
\hline
\end{tabular}




\section{A.4.3 BIBB - Trending - Automated Trend Retrieval - A (T-ATR-A)}

The A device notifies the B device that a trending buffer has acquired a predetermined number of data samples.

\begin{tabular}{|l|c|c|}
\hline \multicolumn{1}{|c|}{ BACnet Service } & Initiate & Execute \\
\hline ConfirmedEventNotification & $\mathrm{x}$ & \\
\hline ReadRange & & $\mathrm{x}$ \\
\hline
\end{tabular}

Devices claiming conformance to T-ATR-A must support the Trend Log object.

\section{A.4.4 BIBB - Trending - Automated Trend Retrieval - B (T-ATR-B)}

The $\mathrm{B}$ device responds to a notification that a trend $\log$ is ready with new data and acquires the new data from the $\log$.

\begin{tabular}{|l|c|c|}
\hline \multicolumn{1}{|c|}{ BACnet Service } & Initiate & Execute \\
\hline ConfirmedEventNotification & & $\mathrm{x}$ \\
\hline ReadRange & $\mathrm{x}$ & \\
\hline
\end{tabular}

\section{A.5 Device Management BIBBs}

These BIBBs prescribe the BACnet capabilities required to interoperably perform the device management functions enumerated in 3.5 for the BACnet devices defined therein.

\section{A.5.1 BIBB - Device Management - Dynamic Device Binding - A (DM-DDB-A)}

The A device seeks information about device attributes of other devices and interprets device announcements.

\begin{tabular}{|l|c|c|}
\hline \multicolumn{1}{|c|}{ BACnet Service } & Initiate & Execute \\
\hline Who-Is & $\mathrm{x}$ & \\
\hline I-Am & & $\mathrm{x}$ \\
\hline
\end{tabular}




\section{A.5.2 BIBB - Device Management - Dynamic Device Binding - B (DM-DDB-B)}

The B device provides information about it's device attributes and responds to requests to identify itself.

\begin{tabular}{|l|c|c|}
\hline \multicolumn{1}{|c|}{ BACnet Service } & Initiate & Execute \\
\hline Who-Is & & $\mathrm{x}$ \\
\hline I-Am & $\mathrm{x}$ & \\
\hline
\end{tabular}

\section{A.5.3 BIBB - Device Management - Dynamic Object Binding - A (DM-DOB-A)}

The A device seeks address information about objects.

\begin{tabular}{|l|c|c|}
\hline \multicolumn{1}{|c|}{ BACnet Service } & Initiate & Execute \\
\hline Who-Has & $\mathrm{x}$ & \\
\hline I-Have & & $\mathrm{x}$ \\
\hline
\end{tabular}

\section{A.5.4 BIBB - Device Management - Dynamic Object Binding - B (DM-DOB-B) )}

The B device provides address information about its objects upon request.

\begin{tabular}{|l|c|c|}
\hline \multicolumn{1}{|c|}{ BACnet Service } & Initiate & Execute \\
\hline Who-Has & & $\mathrm{x}$ \\
\hline I-Have & $\mathrm{x}$ & \\
\hline
\end{tabular}

\section{A.5.5 BIBB - Device Management - DeviceCommunicationControl - A (DM-DCC-A)}

The A device exercises communication control over the B device.

\begin{tabular}{|c|c|c|}
\hline BACnet Service & Initiate & Execute \\
\hline DeviceCommunicationControl & $\mathrm{x}$ & \\
\hline
\end{tabular}

\section{A.5.6 BIBB - Device Management - DeviceCommunicationControl - B (DM-DCC-B) )}

The B device responds to communication control exercised by the A device.

\begin{tabular}{|c|c|c|}
\hline BACnet Service & Initiate & Execute \\
\hline DeviceCommunicationControl & & $\mathrm{x}$ \\
\hline
\end{tabular}




\section{A.5.7 BIBB - Device Management - PrivateTransfer - A (DM-PT-A) )}

The A device initiates the transmission of non-BACnet data within a BACnet service request. The interpretation of the data is a local matter.

\begin{tabular}{|l|c|c|}
\hline \multicolumn{1}{|c|}{ BACnet Service } & Initiate & Execute \\
\hline ConfirmedPrivateTransfer & $\mathrm{x}$ & \\
\hline UnconfirmedPrivateTransfer & $\mathrm{x}$ & \\
\hline
\end{tabular}

\section{A.5.8 BIBB - Device Management - PrivateTransfer - B (DM-PT-B) )}

The B device processes the non-BACnet data contained in the BACnet service request.

\begin{tabular}{|l|c|c|}
\hline \multicolumn{1}{|c|}{ BACnet Service } & Initiate & Execute \\
\hline ConfirmedPrivateTransfer & & $\mathrm{x}$ \\
\hline UnconfirmedPrivateTransfer & & $\mathrm{x}$ \\
\hline
\end{tabular}

\section{A.5.9 BIBB - Device Management - Text Message - A (DM-TM-A) )}

The A device initiates the transmission of text messages. The interpretation and subsequent processing of the messages is a local matter.

\begin{tabular}{|l|c|c|}
\hline \multicolumn{1}{|c|}{ BACnet Service } & Initiate & Execute \\
\hline ConfirmedTextMessage & $\mathrm{x}$ & \\
\hline UnconfirmedTextMessage & $\mathrm{x}$ & \\
\hline
\end{tabular}

\section{A.5.10 BIBB - Device Management - Text Message - B (DM-TM-B)}

The B device processes the text messages.

\begin{tabular}{|l|c|c|}
\hline \multicolumn{1}{|c|}{ BACnet Service } & Initiate & Execute \\
\hline ConfirmedTextMessage & & $\mathrm{x}$ \\
\hline UnconfirmedTextMessage & & $\mathrm{x}$ \\
\hline
\end{tabular}

\section{A.5.11 BIBB - Device Management - TimeSynchronization - A (DM-TS-A)}

The A device provides time synchronization to B devices. The time parameter contained in the service request contains the date and time as determined by the clock in the device issuing the service request. Normally this will be "local time," i.e., the time in the local time zone corrected for daylight savings time as appropriate.

\begin{tabular}{|c|c|c|}
\hline BACnet Service & Initiate & Execute \\
\hline TimeSynchronization & $\mathrm{x}$ & \\
\hline
\end{tabular}

Devices claiming conformance to DM-TS-A must support the Time_Synchronization_Recipients property of the Device object. 


\section{A.5.12 BIBB - Device Management - TimeSynchronization - B (DM-TS-B) )}

The B device interprets time synchronization messages from the A device.

\begin{tabular}{|c|c|c|}
\hline BACnet Service & Initiate & Execute \\
\hline TimeSynchronization & & $\mathrm{x}$ \\
\hline
\end{tabular}

Devices claiming conformance to DM-TS-B must support the Local_Time and Local_Date properties of their Device objects.

\section{A.5.13 BIBB - Device Management - UTCTimeSynchronization - A (DM-UTC-A) )}

The A device provides time synchronization to B devices. The time parameter contained in the service request contains "Coordinated Universal Time" (UTC). For all practical purposes, UTC is synonymous with Greenwich Mean Time, the time at the zero or Greenwich meridian.

\begin{tabular}{|c|c|c|}
\hline BACnet Service & Initiate & Execute \\
\hline UTCTimeSynchronization & $\mathrm{x}$ & \\
\hline
\end{tabular}

Devices claiming conformance to DM-TS-A must support the Time_Synchronization_Recipients property of the Device object.

\section{A.5.14 BIBB - Device Management - UTCTimeSynchronization - B (DM-UTC-B) )}

The B device interprets time synchronization messages from the A device.

\begin{tabular}{|c|c|c|}
\hline BACnet Service & Initiate & Execute \\
\hline UTCTimeSynchronization & & $\mathrm{x}$ \\
\hline
\end{tabular}

Devices claiming conformance to DM-TS-B must support the Local_Time, Local_Date, UTC_Offset, and Daylight_Savings_Status properties of the Device object.

\section{A.5.15 BIBB - Device Management - ReinitializeDevice - A (DM-RD-A) )}

The A device is authorized to reinitialize the B device.

\begin{tabular}{|c|c|c|}
\hline BACnet Service & Initiate & Execute \\
\hline ReinitializeDevice & $\mathrm{x}$ & \\
\hline
\end{tabular}

Devices claiming conformance to DM-RD-A shall be able to initiate ReinitializeDevice requests containing the Password parameter. This shall be both for warm and cold start. 


\section{A.5.16 BIBB - Device Management - ReinitializeDevice - B (DM-RD-B)}

The B device performs reinitialization requests from the A device. The optional password field shall be supported.

\begin{tabular}{|c|c|c|}
\hline BACnet Service & Initiate & Execute \\
\hline ReinitializeDevice & & $\mathrm{x}$ \\
\hline
\end{tabular}

\section{A.5.17 BIBB - Device Management - Backup and Restore - A (DM-BR-A)}

The A device uploads a file which contains the configuration of the B device and downloads that file to the B device should it need to be restored to its previously backed-up state.

\begin{tabular}{|l|c|c|}
\hline \multicolumn{1}{|c|}{ BACnet Service } & Initiate & Execute \\
\hline AtomicReadFile & $\mathrm{x}$ & \\
\hline AtomicWriteFile & $\mathrm{x}$ & \\
\hline
\end{tabular}

Devices claiming conformance to DM-BR-A must write the value TRUE to the Archive property of the File object which represents the configuration file in the B device following a backup operation. Configuration file uploads and downloads shall be performed using stream-oriented file access.

\section{A.5.18 BIBB - Device Management - Backup and Restore - B (DM-BR-B) )}

The B device provides its configuration file to the A device and allows the A device to download this file to recover its configuration in the event of a failure.

\begin{tabular}{|l|c|c|}
\hline \multicolumn{1}{|c|}{ BACnet Service } & Initiate & Execute \\
\hline AtomicReadFile & & $\mathrm{x}$ \\
\hline AtomicWriteFile & & $\mathrm{x}$ \\
\hline
\end{tabular}

In devices claiming conformance to DM-BR-B, the Read_Only property of File objects which represent configuration files shall contain the value FALSE, the File_Type property shall contain the value CONFIGURATION, and the File_Size property shall be writable. Configuration file uploads and downloads shall be performed using stream-oriented file access.

\section{A.5.19 BIBB - Device Management - List Manipulation - A (DM-LM-A)}

Many BACnet object types have properties that are lists of a particular datatype. The A device can add and remove list elements in properties of objects in the B device.

\begin{tabular}{|l|c|c|}
\hline \multicolumn{1}{|c|}{ BACnet Service } & Initiate & Execute \\
\hline AddListElement & $\mathrm{x}$ & \\
\hline RemoveListElement & $\mathrm{x}$ & \\
\hline
\end{tabular}




\section{A.5.20 BIBB - Device Management - List Manipulation - B (DM-LM-B)}

The B device responds to requests to add or remove list elements.

\begin{tabular}{|l|c|c|}
\hline \multicolumn{1}{|c|}{ BACnet Service } & Initiate & Execute \\
\hline AddListElement & & $\mathrm{x}$ \\
\hline RemoveListElement & & $\mathrm{x}$ \\
\hline
\end{tabular}

\section{A.5.21 BIBB - Device Management - Object Creation and Deletion - A (DM-OCD-A)}

BACnet allows object instances to be dynamically created and deleted. The A device may dynamically create and delete the object types supported by the B device.

\begin{tabular}{|l|c|c|}
\hline \multicolumn{1}{|c|}{ BACnet Service } & Initiate & Execute \\
\hline CreateObject & $\mathrm{x}$ & \\
\hline DeleteObject & $\mathrm{x}$ & \\
\hline
\end{tabular}

\section{A.5.22 BIBB - Device Management - Object Creation and Deletion - B (DM-OCD-B)}

The B device creates and deletes object instances based on requests from the A device. The object types whose dynamic creation and deletion is supported shall be enumerated in the $\mathrm{B}$ device's PICS.

\begin{tabular}{|l|c|c|}
\hline \multicolumn{1}{|c|}{ BACnet Service } & Initiate & Execute \\
\hline CreateObject & & $\mathrm{x}$ \\
\hline DeleteObject & & $\mathrm{x}$ \\
\hline
\end{tabular}

\section{A.6 Network Management BIBBs}

These BIBBs prescribe the BACnet capabilities required to interoperably perform the network management functions enumerated in 3.5 for the BACnet devices defined therein.

\section{A.6.1 BIBB - Network Management - Connection Establishment - A (NM-CE-A)}

The A device commands a half-router to establish and terminate connections as needed for communication with other devices.

\begin{tabular}{|l|c|c|}
\hline \multicolumn{1}{|c|}{ BACnet Network Layer Message } & Initiate & Execute \\
\hline Establish-Connection-To-Network & $\mathrm{x}$ & \\
\hline Disconnect-Connection-To-Network & $\mathrm{x}$ & \\
\hline
\end{tabular}




\section{A.6.2 BIBB - Network Management - Connection Establishment - B (NM-CE-B)}

The B device executes commands to establish and terminate connections as needed.

\begin{tabular}{|c|c|c|}
\hline BACnet Network Layer Message & Initiate & Execute \\
\hline Establish-Connection-To-Network & & $\mathrm{x}$ \\
\hline Disconnect-Connection-To-Network & & $\mathrm{x}$ \\
\hline
\end{tabular}

\section{A.6.3 BIBB - Network Management - Router Configuration - A (NM-RC-A)}

The A device may query and change the configuration of routers and half-routers. Note that the capabilities of routers and half-routers are defined in BACnet Clause 6. Thus no explicit BIBBs are required.

\begin{tabular}{|l|c|c|}
\hline \multicolumn{1}{|c|}{ BACnet Network Layer Message } & Initiate & Execute \\
\hline Who-Is-Router-To-Network & $\mathrm{x}$ & \\
\hline I-Am-Router-To-Network & & $\mathrm{x}$ \\
\hline I-Could-Be-Router-To-Network & & $\mathrm{x}$ \\
\hline Initialize-Routing-Table & $\mathrm{x}$ & \\
\hline Initialize-Routing-Table-Ack & & $\mathrm{x}$ \\
\hline
\end{tabular}

\section{A.7 Virtual Terminal BIBBs}

Virtual terminal services permit "remote console" access of field devices from across a BACnet LAN. The effect is to enable access to proprietary configuration and diagnostic routines from a workstation as if the workstation were directly connected to the field device in the mechanical room.

\section{A.7.1 BIBB - Virtual Terminal - A (VT-A)}

The A device initiates a virtual terminal session and exchanges data with the B device.

\begin{tabular}{|l|c|c|}
\hline \multicolumn{1}{|c|}{ BACnet Service } & Initiate & Execute \\
\hline VT-Open & $\mathrm{x}$ & \\
\hline VT-Close & $\mathrm{x}$ & $\mathrm{x}$ \\
\hline VT-Data & $\mathrm{x}$ & $\mathrm{x}$ \\
\hline
\end{tabular}




\section{A.7.2 BIBB - Virtual Terminal - B (VT-B) )}

The B devices permits the establishment of a virtual terminal session and exchanges data with the A device.

\begin{tabular}{|l|c|c|}
\hline \multicolumn{1}{|c|}{ BACnet Service } & Initiate & Execute \\
\hline VT-Open & & $\mathrm{x}$ \\
\hline VT-Close & $\mathrm{x}$ & $\mathrm{x}$ \\
\hline VT-Data & $\mathrm{x}$ & $\mathrm{x}$ \\
\hline
\end{tabular}




\section{Annex B. Descriptions and Profiles of Standardized BACnet Devices}

This clause provides descriptions of five "standardized" types of BACnet devices. Any device that implements all the required BACnet capabilities for a particular device type and interoperability area may claim to be a device of that particular type. Devices may also provide additional capabilities and shall indicate these capabilities in their PICS. The devices defined herein are: BACnet Operator Workstation, BACnet Building Controller, BACnet Advanced Application Controller, BACnet Application Specific Controller, BACnet Smart Actuator, and BACnet Smart Sensor.

\section{B.1 BACnet Operator Workstation (B-OWS)}

The B-OWS is the operator's window into a BACnet system. While it is primarily used for the operation of a system, it may be used for configuration activities that are beyond the scope of this standard. It is not intended to perform direct digital control. It enables the specification of the following:

Data Sharing

- Archival storage of data

- Presentation of data (i.e., reports and graphics)

- The ability to monitor the value of all BACnet object types, including all required and optional properties

- The ability to modify setpoints and parameters

Alarm and Event Management

- Operator notification and presentation of event information

- Alarm acknowledgment by operators

- Alarm summarization

- Adjustment of alarm limits

- Adjustment of alarm routing

Scheduling

- Modification of schedules

- Display of the start and stop times (schedule) of scheduled devices

Trending

- Modification of the parameters of a trend log

- Display and archive of trend data 
Device and Network Management

- Display of information about the status of any device on the BACnet inter-network

- Display of information about any object in the BACnet inter-network

- Ability to silence a device on the network that is transmitting erroneous data

- Ability to synchronize the time in devices across the BACnet inter-network

- Ability to cause a remote device to reinitialize itself

- Ability to backup and restore the configuration of other devices

- Ability to command half-routers to establish and terminate connections

- Ability to query and change the configuration of half-routers and routers

\section{B.2 BACnet Building Controller (B-BC)}

A B-BC is a general purpose, field programmable device capable of carrying out a variety of building automation and control tasks. It enables the specification of the following:

Data Sharing

- Ability to provide the values of any of its BACnet objects

- Ability to retrieve the values of BACnet objects from other devices

- Ability to allow modification of some or all of its BACnet objects by another device

Alarm and Event Management

- Generation of alarm / event notifications and the ability to direct them to recipients

- Maintain a list of unacknowledged alarms / events

- Notifying other recipients that the acknowledgment has been received

- Adjustment of alarm / event parameters

Scheduling

- Ability to schedule output actions, both in the local device and in other devices, both binary and analog, based on date and time

Trending

- Collection and delivery of (time, value) pairs.

Device and Network Management

- Ability to respond to information about its status

- Ability to respond to requests for information about any of its objects

- Ability to respond to communication control messages

- Ability to synchronize its internal clock upon request

- Ability to perform re-initialization upon request

- Ability to upload its configuration and allow it to be subsequently restored

- Ability to command half-routers to establish and terminate connections 


\section{B.3 BACnet Advanced Application Controller (B-AAC)}

A B-AAC is a control device with limited resources relative to a B-BC. It may be intended for specific applications and supports some degree of programmability.

Data Sharing:

- Ability to provide values for any of its BACnet objects upon request

- Ability to allow modification of some or all of its BACnet objects by another BACnet device

Alarm and Event Management

- Generation of limited alarm and event notifications and the ability to direct them to recipients

- Tracking acknowledgements of alarms from human operators

- Adjustment of alarm parameters

Scheduling

- Ability to schedule actions in the local device based on date and time

Trending

- No requirement

Device and Network Management

- Ability to respond to queries about its status

- Ability to respond to requests for information about any of its objects

- Ability to respond to communication control messages

- Ability to synchronize its internal clock upon request

- Ability to perform re-initialization upon request

\section{B.4 BACnet Application Specific Controller (B-ASC)}

A B-ASC is a controller with limited resources relative to a B-AAC. It is intended for use in a specific application and supports limited programmability. It enables specification of the following:

Data Sharing

- Ability to provide the values of any of its BACnet objects

- Ability to allow modification of some or all of its BACnet objects by another device

Alarm and Event Management

- None

Scheduling

- None

Trending

- None 
Device and Network Management

- Ability to respond to information about its status

\section{B.5 BACnet Smart Actuator (B-SA)}

A B-SA is a simple control device with limited resources, intended for specific applications.

Data Sharing:

- Ability to provide values for any of its BACnet objects upon request

- Ability to allow modification of some or all of its BACnet objects by another device

Alarm and Event Management

- No requirement

Scheduling

- No requirement

Trending

- No requirement

Device and Network Management

- No requirement

\section{B.6 BACnet Smart Sensor (B-SS)}

An SS is a simple sensing device with very limited resources.

Data Sharing:

- Ability to provide values for any of its BACnet objects upon request

Alarm and Event Management

- No requirement

Scheduling

- No requirement

Trending

- No requirement

Device and Network Management

- No requirement 


\section{B.7 BACnet Gateway (B-GW)}

A B-GW is a device that provides bi-directional translation of data and information between BACnet devices and devices that communicate using a non-BACnet communication protocol. It enables specification of the following:

Data Sharing:

- Ability to provide values for any point on the non-BACnet side of the gateway to BACnet devices as if the values were originating from BACnet objects

- Ability to allow BACnet devices to modify some or all of the point values on the non-BACnet side of the gateway by using standard BACnet write services

Alarm and Event Management

- Generation of alarm / event notifications and the ability to direct them to recipients

- Maintenance of a list of unacknowledged alarms / events

- Notification of other recipients that an acknowledgment has been received

- Adjustment of alarm / event parameters

Scheduling

- Ability to schedule output actions, both in the local device and in other devices, both binary and analog, based on date and time

Trending

- Collection and delivery of (time, value) pairs

Device and Network Management

- Ability to respond to information about its status

- Ability to respond to requests for information about any of its objects

- Ability to respond to communication control messages

- Ability to synchronize its internal clock upon request

- Ability to perform re-initialization upon request 


\section{B.8 Profiles of the Standard BACnet Devices}

The following tables indicate which BIBBs must be supported by each device type for each interoperability area.

\begin{tabular}{|c|c|c|c|c|c|c|c|}
\hline & B-OWS & B-BC & B-AAC & B-ASC & B-SA & B-SS & B-GW \\
\hline Data Sharing & DS-RP-A,B & DS-RP-A,B & DS-RP-B & DS-RP-B & DS-RP-B & DS-RP-B & DS-RP-B \\
\hline & DS-RPM-A & DS-RPM-A,B & DS-RPM-B & DS-WP-B & DS-WP-B & & DS-RPM-B \\
\hline & DS-WP-A & DS-WP-A,B & DS-WP-B & & & & DS-WP-B \\
\hline & DS-WPM-A & DS-WPM-B & DS-WPM-B & & & & DS-WPM-B \\
\hline & & DS-COVU-A,B & & & & & \\
\hline
\end{tabular}

\begin{tabular}{|c|c|c|c|c|c|c|c|}
\hline & B-OWS & B-BC & B-AAC & B-ASC & B-SA & B-SS & B-GW \\
\hline Alarm \& Event & AE-N-A & AE-N-B & AE-N-B & & & AE-N-B \\
\hline Mgmt & AE-ACK-A & AE-ACK-B & AE-ACK-B & & & & AE-ACK-B \\
\hline & AE-ASUM-A & A-ASUM-B & AE-ASUM-B & & & & A-ASUM-B \\
\hline & AE-ESUM-A & AE-ESUM-B & & & & & AE-ESUM-B \\
\hline
\end{tabular}

\begin{tabular}{|c|c|c|c|c|c|c|c|}
\hline & B-OWS & B-BC & B-AAC & B-ASC & B-SA & B-SS & B-GW \\
\hline Scheduling & SCHED-A & SCHED-B & SCHED-B & & & & SCHED-B \\
\hline
\end{tabular}

\begin{tabular}{|c|c|c|c|c|c|c|c|}
\hline & B-OWS & B-BC & B-AAC & B-ASC & B-SA & B-SS & B-GW \\
\hline Trending & T-VMT-A ${ }^{*}$ & T-VMT-B ${ }^{*}$ & & & & & T-VMT-B $^{*}$ \\
\hline & T-ATR-A & T-ATR-B & & & & & \\
\hline
\end{tabular}

\begin{tabular}{|c|c|c|c|c|c|c|c|}
\hline & B-OWS & B-BC & B-AAC & B-ASC & B-SA & B-SS & B-GW \\
\hline Device \& & DM-DDB-A,B & DM-DDB-A,B & DM-DDB-B & DM-DDB-B & & & DM-DDB-B \\
\hline Network Mgmt & DM-DOB-A,B & DM-DOB-A,B & DM-DOB-B & DM-DOB-B & & & DM-DOB-B \\
\hline & DM-DCC-A & DM-DCC-B & DM-DCC-B & DM-DCC-B & & & DM-DCC-B \\
\hline & DM-TS-A & $\begin{array}{c}\text { DM-TS-B } \\
\text { or } \\
\end{array}$ & $\begin{array}{c}\text { DM-TS-B } \\
\text { or } \\
\text { DM-UTC-B }\end{array}$ & & & DM-TS-B \\
DM-UTC-B & & & & or \\
& & & & & & DM-UTC-B \\
\hline & DM-UTC-A & & & & & DM-RD-B \\
\hline & DM-RD-A & DM-RD-B & DM-RD-B & & & & \\
\hline & DM-BR-A & DM-BR-B & & & & & \\
\hline
\end{tabular}

* At the time of publication of this guide the BACnet services required to implement these BIBBs have not completed the public review process for becoming part of the BACnet standard. 


\section{Annex C. GSA BACnet Implementation Checklist}

The purpose of this Annex is to provide a simple checklist to ensure that the most important elements of a successful BACnet building automation and control system have been considered and specified as needed.

\section{General}

Have the functional requirements of the job, including all sequences of operation, been thoroughly specified?

Have all the desired workstation capabilities, features, and characteristics been specified including:

\section{Graphics}

Tabular reports

Trend $\operatorname{logs}$

Operator tools

Security levels and privileges

Have the desired local or wide area network technologies been specified? If communication via an IP internet is required, has the use of BACnet Tunneling Routers or BACnet/IP been specified?

Has BACnet been specified for all levels of the control system hierarchy using devices conforming to the profiles in Annex B?

\section{Data Sharing (3.1)}

\section{$\underline{\text { Point list }}$}

Have point lists been compiled showing each sensor, actuator, setpoint, and other parameters that are to be accessible over the network?

\section{Presentation of data}

Has the format and desired content of each tabular report been specified?

Have the desired graphic displays been defined along with the maximum allowable update interval?

Has it been specified that all points in the system must be available for real-time plotting at user-definable update intervals and that multiple analog and binary values may be plotted on the same axes? 
Monitoring of any property of any BACnet object type

Has it been specified that it must be possible to read and display the value of any property of any object in the system?

\section{Global objects}

Have all shared global data points been specified?

\section{$\underline{\text { Setpoint and parameter modification }}$}

Has it been specified which operating setpoints and parameters must be available for modification via BACnet services (as opposed to proprietary vendor-specific means)?

Have the desired means of making the modifications, e.g., via a graphical user interface (GUI), been specified?

\section{Peer-to-Peer data exchange}

Have any required data dependencies (interlocks, shared setpoints, schedules, and so on) that must be implemented via the network been defined?

\section{Alarm and Event Management (3.2)}

\section{Alarm lists, operator notification and presentation of event information}

Have all desired alarms, including alarm limits, interlocks to avoid "nuisance" alarms, and desired response time from the occurrence of an event until a notification is provided been specified?

Has it been specified how alarms are to be categorized and distributed, i.e., which alarms go where and when?

Has it been specified how alarms are to appear at the operator workstation?

Has it been specified that "intrinsic reporting" shall be used when it is sufficient to meet the functional requirements?

\section{Alarm acknowledgment}

Has it been specified that alarm acknowledgment capability be provided and that a log be maintained that records when an alarm notification was received, when it was acknowledged, and by whom? 


\section{Alarm summarization}

Has it been specified that it shall be possible for an operator to receive, at any time, a summary of all alarms that are currently in effect and whether or not they have been acknowledged?

Has it been specified that is shall be possible to receive a summary of all alarms regardless of acknowledgment status; for which a particular recipient is enrolled for notification; based on current event state; based on the particular BACnet event algorithm (e.g., change of value, change of state, out of range, and so on); alarm priority; and notification class?

\section{$\underline{\text { Alarm parameter adjustment }}$}

Has it been specified that operators shall be provided the capability to dynamically modify the alarm parameters for all standard BACnet event types?

\section{$\underline{\text { Alarm routing adjustment }}$}

Has it been specified that operators shall have the ability to change alarm routing for each alarm including the destination for each type of alarm and alarm priority, the day of week and time of day, and the type of transition involved (TO-OFFNORMAL, TO-NORMAL and so on)?

\section{Scheduling (3.3)}

$\underline{\text { Schedule list }}$

Has each action that should take place based on the date and time of day been specified?

Display of start and stop times of scheduled devices

Has it been specified that an operator shall be able to inspect the content of any schedule and determine the specific control actions that will occur at any time, on any date? Additionally, for any particular device or system parameter that is the subject of a schedule, has it been specified that an operator shall be able to determine the schedule of actions related to that device or parameter?

\section{Modification of schedules}

Has it been specified that certain, or all, calendar entries and schedules shall be modifiable from an operator workstation?

\section{Trending (3.4)}

\section{Archival storage of data}

Has the maximum number of data points for which archival storage is needed, along with the minimum sampling interval been specified? 
Has the duration of time for which the archived information must be available for on-line retrieval been specified?

Has the means for archiving older data, e.g., tape or CD-R, been specified?

\section{$\underline{\text { Trend list }}$}

Have the initial requirements for trending in terms of which data points are to be trended, the sampling rate, the duration of each trend log, and the length of time it is desired to keep the resulting logs available on-line been specified? Alternatively, have the approximate number of desired trend logs been specified?

Display and archive of trend data

Has it been specified that an operator will be able to retrieve and display trend logs, access the underlying numerical data in spreadsheet format, and output the data to printers or other files?

\section{Modification of trend log parameters}

Has it been specified that the data points to be logged, the sampling rate, and duration of trend logs shall be modifiable by an authorized operator from a system workstation?

\section{Device and Network Management (3.5)}

Display of information about device status

Has it been specified that an operator shall be able to display at any time the operational status of any device on the BACnet internetwork?

Display of information about any BACnet object

Has it been specified that an operator shall be able to display at any time any property of any BACnet object?

Has it been specified that an operator shall also be able to display property values of objects grouped by object type, object location, and building system?

Ability to silence a device on the network that is transmitting erroneous data

Has it been specified that an operator shall be able to direct a field device to stop transmitting event or alarm notifications until a subsequent command to resume transmissions is received? 
Ability to synchronize the time in devices across the BACnet inter-network

Has it been specified that an operator shall be able to set the time and date in any device on the network that supports time-of-day functionality? This capability should be required for both individual devices or groups of devices, including all devices simultaneously.

Ability to cause a remote device to reinitialize itself

Has it been specified that an operator shall have the ability to issue reinitialization commands to any device that supports remote reinitialization?

Ability to backup and restore the configuration of other devices

Has it been specified that an operator shall have the ability to backup and restore all BACnet devices on the network that support this capability?

Ability to command half-routers to establish and terminate connections

Has it been specified that an operator shall have the ability to issue a command for the establishment or termination of a connection to a remote BACnet network?

Ability to query and change the configuration of half-routers and routers

Has it been specified that an operator shall have the ability to display and modify the routing table entries in all provided BACnet half-routers and routers?

\section{Use of BACnet Objects (4)}

\section{Naming Conventions (4.1)}

Has a set of abbreviations for common building systems, subsystems, and points that are to be used by all vendors been specified?

Has it been specified that object name properties, in those devices where the object name is configurable, shall be at least 50 characters long and shall, to the extent possible make use of a system and point nomenclature using the abbreviations specified above?

Has it been specified that BACnet object names used in workstations may be up to 50 characters long and shall consist of a string made up of components indicating, as appropriate, the location, system, subsystem, and point of the object? Also, that these object names shall be used in workstation applications such as graphics, reports, and alarms wherever an object name is appropriate in lieu of the object name property in the remote device? Also, that an operator may display at any time the mapping between the object name used on the workstation and the object name property used in the remote BACnet device? 
Commissioning/Diagnostic Mode (4.2)

Has it been specified that the Out_Of_Service property shall be adjustable (writable) using BACnet services for all Analog, Binary, Multi-state, Loop and Program objects?

\section{Using Object Descriptions (4.3)}

Has it been specified that each Device object shall have a Description property and that the length available for the Description properties of other implemented object types shall be provided in the "Property Range Restrictions" portion of the device's PICS?

\section{Issues Related to Specific BACnet Object Types (4.4)}

Analog Input, Output, and Value (4.4.1)

Has it been specified that all analog objects (Input, Output, and Value) shall have the capability of using the Change of Value reporting mechanism and that the COV_Increment property shall be writable using BACnet services?

\section{Binary Input (4.4.2)}

Has it been specified, for each Binary Input object, the text to be used for the Inactive_Text and Active_Text properties?

\section{Binary Output (4.4.3)}

Has it been specified, for each Binary Input object, the text to be used for the Inactive_Text and Active_Text properties? In addition, if appropriate for the application, have appropriate values for the Feedback_Value, Minimum_On_Time, and Minimum_Off_Time properties been specified?

Has support for the Change_Of_State_Time, Change_Of_State_Count, and Time_Of_State_Count_Reset been specified if counting state changes is appropriate for the application? If so, has it also been specified that Change_Of_State_Count be writable so that the count can be reset?

Has support for the Elapsed_Active_Time and Time_Of_Active_Time_Reset properties been specified if accumulating run time is appropriate for the application? If so, has it also been specified that Elapsed_Active_Time be writable so that the run time can be reset?

\section{Binary Value (4.4.4)}

Has it been specified, for each Binary Value object, the text to be used for the Inactive_Text and Active_Text properties? In addition, if appropriate for the application, have appropriate values for the Minimum_On_Time, and Minimum_Off_Time properties been specified? 
Has support for the Change_Of_State_Time, Change_Of_State_Count, and Time_Of_State_Count_Reset been specified if counting state changes is appropriate for the application? If so, has it also been specified that Change_Of_State_Count be writable so that the count can be reset?

Has support for the Elapsed_Active_Time and Time_Of_Active_Time_Reset properties been specified if accumulating run time is appropriate for the application? If so, has it also been specified that Elapsed_Active_Time be writable so that the run time can be reset?

\section{$\underline{\text { Calendar (4.4.5) }}$}

Has it been specified that devices that provide scheduling capability shall also provide at least one Calendar object with a capacity of at least 10 entries and that it shall be possible to view the Calendar object and make modifications from any BACnet workstation on the network?

Has it been specified, if the Calendar's Date_List property is writable using BACnet services, that all calendar entry datatypes must be supported?

\section{$\underline{\text { Loop (4.4.6) }}$}

Has it been specified that the desired PID control loops shall be represented by Loop objects and that the tuning constant properties shall be writable? Has writability of other Loop properties been specified as appropriate to the application?

Has it been specified that all Loop objects shall have the capability of using the Change of Value reporting mechanism and that the COV_Increment property shall be writable using BACnet services?

\section{Multi-state Input, Output, and Value (4.4.7)}

Has it been specified, for each Multi-state Input, Output, and Value object, the text to be used for each state that the object can represent? In addition, if appropriate for the application, has it also been specified how the Feedback_Value is to be determined?

Has it been specified that all Multi-state Input, Output, and Value objects shall have the capability of using the Change of Value reporting mechanism and that the COV_Increment property shall be writable using BACnet services?

Schedule (4.4.8)

Has each building system that is to be subjected to date and time scheduling been specified and that it shall be possible to modify schedule entries from a BACnet workstation?

\section{Dynamic Object Creation (4.5)}

Has it been specified, if required by the application, that it shall be possible to dynamically create instances of the Averaging, Calendar, Event Enrollment, Group, Notification Class, Schedule, and Trend Log objects? 


\section{Use of BACnet Services (5)}

Interoperable Commands (5.1)

Have the processes that are to be prioritized and the command priority levels assigned to each if they do not fall into the pre-assigned levels shown in Table 1 been specified? For each point subject to the command priority scheme, has a default status, position, or value for the point to take on in the absence of a prioritized command been specified?

\section{Assigning Alarm Priorities (5.2.1)}

Have the priority values to be assigned for each alarm in the system been specified? For each priority value, or range of values, have the actions to be taken upon receipt been specified?

\section{Setting up Notification Classes (5.2.2)}

Has it been specified how alarms should be distributed by specifying the recipients of each type and priority of alarm? If desired, have the valid days of the week and times of the day for each been specified?

\section{Event Notification Message Texts (5.2.3)}

Have the content and format of the alarm messages that will be delivered to operators been specified?

\section{Assigning Levels of Authority to Certain Operators (5.3)}

Has it been specified, if desired, the number of authorization levels and the operator accounts to be assigned to each? If password protection for remote device management services is needed, have the passwords to be configured been specified? Alternatively, has it been specified that there shall be a method provided for dynamically assigning passwords for remote device management functions after installation?

\section{$\underline{\text { Subscribed COV Notifications (5.4.1) }}$}

Has it been specified that workstation software shall be provided that has the capability of subscribing to COV notifications for all object types that support it?

\section{Unsubscribed COV Notifications (5.4.2)}

Has it been specified that changes of value of globally shared data shall be distributed by means of UnconfirmedCOVNotifications?

\section{Time Synchronization (5.5)}

Has it been specified that a time master shall be provided along with the format of the time synchronization messages, either local time or UTC? 


\section{Specifying System/Network Architecture (6)}

\section{System Architectures (6.1)}

Has it been specified that BACnet shall be used throughout the building automation and control internetwork at all levels, i.e., that the system shall be "native" BACnet?

Has it been specified that the internetwork shall be configured such that, if three or more networks are involved with different performance characteristics, the faster networks shall be used to interconnect the slower ones?

\section{Local Area Network Selection (6.2)}

\section{$\underline{\text { ISO 8802-3, Ethernet (6.2.1) }}$}

Has it been specified which devices in the control system should reside on the Ethernet LAN?

Has the transmission medium option that is to be used been specified? If more than one will be used, has it been specified which one is to be used where, and the devices that are to be connected? If hubs will be needed, have the number of ports and the kind of medium for each port been specified?

Has the Ethernet speed (10 Mbps or $100 \mathrm{Mbps})$ been specified?

\section{ANSI/ATA 878.1, ARCNET (6.2.2)}

Has it been specified which devices in the control system should reside on the ARCNET LAN?

Has the transmission medium option that is to be used been specified? If more than one will be used, has it been specified which one is to be used where and the devices that are to be connected? If hubs will be needed, have the number of ports and the kind of medium for each port been specified?

Has it been specified how ARCNET addresses are to be assigned?

\section{Master-Slave/Token-Passing, MS/TP (6.2.3)}

Has it been specified which devices in the control system should reside on the MS/TP LAN?

Has the baud rate to be used been specified?

Has it been specified how MS/TP addresses are to be assigned and, if slave devices will be used, how the address space is to be divided between slaves and masters? 
EIA-709.1, LonTalk (6.2.4)

Has it been specified which devices in the control system should reside on the LonTalk LAN?

Have the media, speed and topology to be used been specified?

Has it been specified how LonTalk addresses are to be assigned?

\section{$\underline{\text { Point-to-Point, PTP (6.2.5) }}$}

Has it been specified that PTP shall be used as the means to attach to a BACnet LAN via a dial-up connection and that the optional password protection shall be provided?

\section{Specifying MAC Addresses in a Consistent Manner (6.3)}

Has the GSA, the design engineer, or the system integrator determined how MAC addresses are to be assigned for each network in the proposed BACnet internetwork using the recommendations in 6.3 ?

\section{Network Numbering Conventions (6.4)}

Has the GSA, the design engineer, or the system integrator determined how network numbers are to be assigned for each network in the proposed BACnet internetwork using the recommendations in 6.4 ?

\section{Device Object Identifier Conventions (6.5)}

Has the GSA, the design engineer, or the system integrator determined how device object identifiers are to be assigned for each device in the proposed BACnet internetwork using the recommendations in 6.5 ?

\section{Use of BACnet with the Internet Protocols (6.6)}

Has it been specified, if existing BACnet non-IP devices are to be interconnected via an IP network, that BACnet Tunneling Routers conforming to Annex H shall be provided?

Has it been specified, for new networks which need IP connectivity, that devices shall be provided that conform to Addendum 135a, BACnet/IP and that, for broadcast distribution, BBMDs shall be provided or appropriate arrangements made for the use of IP multicasting?

\section{Routers - Interconnecting Multiple BACnet Networks (6.7)}

Has it been specified that it shall be the contractor's responsibility to configure each router using the network numbering scheme agreed to for the project?

Has it been specified that each router shall be configured such that all network layer error messages shall be directed to a specific workstation using the BACnet 
ConfirmedTextMessage service? Has the GSA, the design engineer, or the system integrator determined which workstation that shall be?

Has it been specified that it shall be the contractor's responsibility to initially configure each router with routing tables containing all network numbers that are part of the project's internet?

Has it been specified that the router shall be able to receive messages at each port of any length that is valid for the LAN technology connected to that port, and to forward the message to any directly-connected network that can convey a message of that size?

\section{Message Segmentation (6.8)}

Has it been specified that all BACnet devices shall support the reception of messages up to the largest size permitted by the LAN technology or support reception of segmented messages?

Has it been specified that all BACnet devices shall support the transmission of messages up to the largest size permitted by the LAN technology or support transmission of segmented messages?

Has it been specified that all workstations shall support both segmented message transmission and segmented message reception?

Has it been specified that all BACnet Building Controllers (see B.2) shall support both segmented message transmission and segmented message reception?

\section{Gateways - Connecting to Legacy Systems (6.9)}

Has it been specified that the operator workstation shall display information from both the BACnet and non-BACnet devices? If appropriate, has it been specified which other devices should also be able to communicate through the gateway?

Has it been specified which way information must flow through the gateway or that the gateway should provide bi-directional communication?

Has it been specified which non-BACnet points are to be readable from the BACnet side of the gateway and which BACnet objects are to be readable from the non-BACnet side of the gateway?

Has it been specified which non-BACnet points are to be modifiable from the BACnet side of the gateway and which BACnet objects are to be modifiable from the non-BACnet side of the gateway?

Has it been specified how much expansion capacity is required in the gateway?

Has it been specified how controller program and configuration database archives are to be created and maintained? 
Have any gateway features that will be needed to support collection of trend data been specified?

Have any gateway features that will be needed to support scheduling been specified?

Have any gateway features that will be needed to support alarm and event handling been specified?

\section{Practical Considerations in the Construction of BACnet Systems (7)}

\section{Procurement Phase (7.1)}

If an RFP is to used, does it require the prospective controls vendor to provide a description of the proposed system containing:

Narrative providing an overview of the BAS system, primary components, workstation(s), and LAN architecture?

Identification of any clarifications or exceptions to the project specifications?

A listing and quantification of all microprocessor-based BAS equipment to be supplied and associated information "cut-sheets" for each?

A listing of references, as appropriate, for projects of a similar nature to the proposed BAS?

The option of requesting a formal BAS demonstration at either the vendor's local offices or customer location?

Construction Phase (7.2)

\section{PICS (7.2.1)}

Has a PICS been provided containing at least:

BACnet Standard Application Services Supported?

Standard Object Types Supported?

Data Link Layer Options?

Special Functionality?

Property Range Restrictions? 


\section{Points Lists (7.2.2)}

Have Points Lists been provided containing at least:

Hardware I/O information?

Proposed I/O Names?

BACnet Object Description?

\section{Record Documentation (7.4.1)}

Before system acceptance, does the GSA have in its possession:

"As-built" record drawings?

I/O Points Lists?

Documentation for any non-standard BACnet objects, properties, or enumerations?

Complete program descriptions of all control and application software per the system installation including English narratives of control/application programs, flowcharts and actual source code/files?

\section{Operator Training (7.4.2)}

Have the minimum number of training hours and course content been specified? 